INAPP WP n. 73

\section{Formazione continua}

e innovazione tecnologica nelle macro-aree italiane: evidenze dall'indagine ROLA

\section{Valentina Ferri}

Domenico Nobili

Giuliana Tesauro

Sergio Ferri

OTTOBRE 2021 


\title{
Formazione continua
}

\section{e innovazione tecnologica nelle macro-aree italiane: evidenze dall'indagine ROLA}

\author{
Valentina Ferri \\ Istituto nazionale per l'analisi delle politiche pubbliche (INAPP), Roma \\ v.ferri@inapp.org
}

Domenico Nobili

Istituto nazionale per l'analisi delle politiche pubbliche (INAPP), Roma

d.nobili@inapp.org

\section{Giuliana Tesauro}

Istituto nazionale per l'analisi delle politiche pubbliche (INAPP), Roma

g.tesauro@inapp.org

\section{Sergio Ferri}

Istituto nazionale per l'analisi delle politiche pubbliche (INAPP), Roma

s.ferri@inapp.org

OTTOBRE 2021

Il presente lavoro nasce dalla convenzione tra Inapp e Fondimpresa. La collaborazione istituzionale avviatasi nel 2018 ha consentito di qualificare e affinare le metodologie, gli strumenti e le attività del Monitoraggio Valutativo finalizzate a valorizzare le esperienze formative finanziate da Fondimpresa. La convenzione ha come oggetto lo studio e l'analisi: dei piani formativi, finanziati dal Fondo tramite il Conto di Sistema e il Conto Formazione; della valutazione d'impatto della formazione sui percorsi professionali dei lavoratori e sulle performance d'impresa, al fine di evidenziarne gli effetti nel Rapporto Nazionale del Monitoraggio Valutativo; dei cambiamenti produttivi e organizzativi derivanti dalla formazione sulle tecnologie abilitanti (Industria 4.0). Tra i principali strumenti di ricerca sviluppati grazie alla collaborazione tra Inapp e Fondimpresa, si annovera I'indagine ROLA (Rilevazione delle opinioni dei lavoratori e delle aziende) giunta ormai alla sua terza edizione.

Gruppo di lavoro Fondimpresa-Inapp: Maurizio Bernava, Nausica lencenelli, Valentina Ferri, Domenico Nobili, Giuliana Tesauro, Sergio Ferri.

Le opinioni espresse in questo lavoro impegnano la responsabilità degli autori e non necessariamente riflettono la posizione dell'Ente di appartenenza.

SOMMARIO: 1. Introduzione. - 2. La formazione come determinante del cambiamento nella letteratura recente. - 3. L'accrescimento della conoscenza attraverso la formazione sulle 'tecnologie abilitanti' nella percezione dei lavoratori coinvolti. - 4. Le determinanti dei cambiamenti percepiti dai lavoratori formati relativamente alle mansioni svolte e agli assetti produttivi e organizzativi. -5 . Conclusioni. - Bibliografia

INAPP - Istituto nazionale per l'analisi delle politiche pubbliche

Corso d'Italia $33 \quad$ Tel. +39 06854471




\title{
ABSTRACT
}

\section{Formazione continua e innovazione tecnologica nelle macro-aree italiane: evidenze dall'indagine ROLA}

\begin{abstract}
Attraverso questo lavoro - basato sull'indagine ROLA (Rilevazione delle opinioni dei lavoratori e delle aziende) - abbiamo potuto osservare come le differenze nell'ambito della formazione siano rilevanti tra Nord, Centro e Sud. Come primo obiettivo abbiamo stimato il livello medio di conoscenze nell'ambito delle innovazioni e la sua correlazione con diverse variabili, tra cui l'aver effettuato o meno un corso di formazione in tali materie. In secondo luogo, abbiamo stimato come l'aver effettuato un corso nell'ambito delle innovazioni tecnologiche si leghi alla probabilità che il lavoratore registri cambiamenti.

Per ciò che riguarda il primo obiettivo, la formazione è sempre associata a un aumento del punteggio in termini di livello medio di conoscenze. Aver svolto il corso di formazione nell'ambito delle innovazioni tecnologiche sembra offrire un apporto molto rilevante nelle aziende. Rispettivamente il livello medio delle conoscenze è associato a un aumento del $19,5 \%$ al Nord, del $20 \%$ al Centro e del 7,8\% al Sud se l'individuo ha effettuato il corso. Per ciò che riguarda la capacità che la formazione ha di tradursi in un cambiamento nelle mansioni dei lavoratori o quantomeno di accompagnare tale tipologia di cambiamento in azienda, osserviamo che i coefficienti più elevati riguardano l'area centrale italiana con 10,6 p.p. e l'area settentrionale con 13,2 p.p., mentre il Sud presenta coefficienti non significativi.
\end{abstract}

PAROLE CHIAVE: formazione professionale continua, fondi interprofessionali per la formazione continua, innovazione tecnologica

JEL CODES: M53, 033, D22 


\section{Introduzione}

Il presente lavoro prende le mosse dai risultati della Rilevazione delle opinioni dei lavoratori e delle aziende (ROLA), avviata nel 2019 nell'ambito delle attività previste dalla Convenzione InappFondimpresa.

L'indagine ROLA ha l'obiettivo di fornire un contributo analitico utile per valutare l'impatto delle iniziative formative promosse e finanziate da Fondimpresa, rilevando le percezioni dei lavoratori formati sia riguardo l'acquisizione di nuove competenze in seguito alla partecipazione alla formazione, sia relativamente ai cambiamenti che questa ha innescato nello svolgimento delle mansioni individuali e nell'organizzazione aziendale. La prima edizione della rilevazione ha interessato un campione di lavoratori coinvolti in formazione nel corso del 2018. Le interviste sono state condotte con metodologia CAWI (Computer Assisted Web Interviewing) utilizzando, a seconda dei casi, strumentazione predisposta dalle aziende, PC personali o dispositivi mobili. Sono state raccolte più di 5.600 interviste complete che hanno consentito di realizzare inferenze ed analisi sull'universo dei formati che, nel 2018, è stato pari a circa 465mila unità con esclusione dei lavoratori delle imprese agricole. L'analisi è stata estesa, con un questionario ad hoc, anche ai responsabili aziendali dei lavoratori coinvolti in formazione.

Allo scopo di realizzare una prima valutazione dell'impatto delle attività formative promosse dal Fondo sulla diffusione dell'innovazione tra le imprese aderenti, l'indagine ROLA riserva una specifica sezione alla rilevazione dei livelli di conoscenza dei formati relativamente a determinati cluster di tecnologie digitali di particolare importanza, coincidenti con le sei tecnologie cosiddette 'abilitanti' individuate e classificate dall'Osservatorio Industria 4.0 del Politecnico di Milano ${ }^{1}$.

Il livello di conoscenza su ciascuno dei sei cluster è stato rilevato per tutti i lavoratori del campione ma con due diverse modalità: ai lavoratori coinvolti in tutte le iniziative formative aventi ad oggetto tematiche connesse con l'innovazione tecnologica è stato richiesto di esprimere le proprie percezioni prima e dopo la formazione; ai lavoratori coinvolti in corsi di formazione centrati su ambiti diversi hanno invece indicato il proprio livello di conoscenza così come percepito al momento della rilevazione.

Utilizzando i dati e le informazioni così ottenute e allo scopo di approfondire le relazioni tra la formazione e i processi evolutivi e trasformativi che da essa scaturiscono, con il presente lavoro si è inteso focalizzare lo sguardo sul diverso grado di efficacia delle iniziative formative riguardanti l'innovazione tecnologica tra le diverse aree del Paese.

Come nell'indagine ROLA, anche in questo specifico approfondimento, l'oggetto prevalente di attenzione saranno le percezioni dei lavoratori formati relativamente all'accrescimento delle

1 Secondo la definizione data dalla Commissione Europea le 'tecnologie abilitanti' sono tecnologie "ad alta intensità di conoscenza e associate ad elevata attività di Ricerca e Sviluppo, a cicli di innovazione rapidi, a consistenti spese d'investimento e a posti di lavoro altamente qualificati". Maggiori dettagli esplicativi circa la natura degli insiemi di 'tecnologie abilitanti', secondo l'accezione qui adottata, sono riportati nel seguente paragrafo 3. 
competenze possedute nei diversi ambiti considerati e ai cambiamenti nelle mansioni svolte e nell'organizzazione del lavoro innescati dalla partecipazione alla formazione.

Fin dalla loro costituzione i Fondi Paritetici Interprofessionali, ed in particolare Fondimpresa (il maggiore per dimensione e capacità finanziaria) hanno sviluppato le attività con una attenzione particolare alle peculiarità del tessuto economico meridionale, spesso attraverso Avvisi ad hoc che, di volta in volta, sono stati diretti verso specifici target di impresa o su particolari tematiche formative. Naturalmente, l'attività dei Fondi nelle regioni del Sud si è svolta nel confronto con un'offerta formativa locale non sempre all'altezza di ogni esigenza e con un livello di diffusione dell'innovazione tecnologica e organizzativa, tra le imprese del territorio, di grado a volte assai inferiore rispetto alle altre aree del Paese. E, come evidenziato nelle pagine seguenti, il ruolo di queste variabili fondamentali, di natura strutturale e non contingente, si rivelerà ancora una volta decisivo nel determinare l'impatto e l'efficacia degli interventi.

\section{La formazione come determinante del cambiamento nella letteratura recente}

L'acquisizione di nuove competenze, indispensabile per lo svolgimento di nuovi compiti e per l'adattamento ai cambiamenti indotti dalla digitalizzazione e dall'automazione è obiettivo prioritario dei percorsi di formazione dei lavoratori. La composizione della forza lavoro è sottoposta ad un mutamento progressivo e crescente con il forte aumento della quota di lavoratori interessati da attività non routinaria rispetto a coloro che svolgono compiti standardizzati (Bachmann et al. 2018). La determinazione della relazione esistente tra la formazione e l'evoluzione delle mansioni individuali e più ancora la misura della sua intensità in un determinato contesto, può esprimere, tra l'altro, un'approssimazione del grado di diffusione delle innovazioni e delle connesse riorganizzazioni aziendali, avviate in vista dell'implementazione di nuovi paradigmi aziendali, collegati all'introduzione delle nuove tecnologie.

In linea generale la realizzazione del potenziale di digitalizzazione richiede sempre una riprogettazione del contenuto dell'attività lavorativa (Brynjolfsson et al. 2018) e spesso, attraverso i nuovi investimenti tecnologici, accanto all'accresciuta domanda dei nuovi profili professionali da inserire in azienda (in Italia in particolare, emerge una crescente necessità di figure tecniche ed esecutive (Ferri e Ricci 2020), si evidenzia sempre un aumento della domanda di formazione. In tal senso, si ritiene di particolare utilità verificare "quanto la formazione possa favorire il processo e se, in ultima analisi, possa essere considerata un effettivo ed operante driver di cambiamento" (Ferri et al. 2017).

La letteratura offre diversi contributi che indagano sugli effetti della formazione e sulla relazione esistente tra questa e i cambiamenti nelle mansioni individuali e negli assetti organizzativi della produzione di beni e servizi. Come ovvio, non rientra tra gli scopi del presente lavoro proporre un'analisi della letteratura in materia ed anche una semplice elencazione che abbia pretese di esaustività richiederebbe ben altro spazio. Tuttavia, anche con l'intento di fornire qualche spunto per ulteriori approfondimenti, può essere utile introdurre qualche cenno ad alcuni recenti contributi connessi più di altri all'argomento del nostro lavoro. Come, ad esempio, il rilevante studio di Tamm (2018) che dimostra come, dopo un periodo di training i lavoratori risultano essere più proficuamente 
coinvolti (e anche coinvolgibili) in attività interattive non routinarie rispetto alla fase precedente la formazione. In questo senso, le diverse tematiche formative determinano un differente impatto sulle mansioni: i contenuti più 'generalisti', come la comunicazione e le soft skills, sono sempre associate allo sviluppo di attività non routinarie. Ancor prima Görlitz e Tamm (2016) avevano indagato la relazione inversa: la stessa partecipazione alla formazione è differente tra i lavoratori con differenti mansioni e coloro che svolgono attività maggiormente routinarie ricevono solitamente meno formazione. Nello stesso lavoro si avanza inoltre un'interessante relazione tra aumento di attività non routinarie e parziale autofinanziamento della formazione con voucher individuale, a ulteriore riprova della più volte osservata maggiore efficacia formativa in connessione con la maggiore responsabilizzazione prodotta dalla partecipazione diretta ai costi.

Il ruolo decisivo delle specificità tematiche viene confermata dagli stimoli che la formazione sui contenuti tecnici conferisce alla attività manuali non di routine, sia dalla sostanziale invarianza che si rileva, per contro, negli esiti formativi di altre iniziative formative particolarmente diffuse (salute e sicurezza, amministrazione, informatica di base). Secondo le conclusioni di Tamm, dunque, la sfida della digitalizzazione che presuppone un incremento delle attività non routinarie, suggerisce la necessità di privilegiare la formazione su tematiche specifiche più suscettibili di produrre impatti e cambiamenti sulle mansioni individuali e migliorare l'adattabilità. Tenendo sempre in conto, naturalmente, le differenze di reazione agli stimoli formativi delle diverse tipologie di utenza, $\mathrm{i}$ lavoratori più scolarizzati e meglio posizionati nella scala gerarchica non solo hanno maggiore accesso alla formazione ma sono già orientati e predisposti ad attività non routinarie. Ciò è ancora è ancora più evidente quando si guarda alla formazione 'autonoma' piuttosto che alla formazione promossa dai datori di lavoro (Görlitz e Tamm 2016).

Il cambiamento delle mansioni può esso stesso innescare un ciclo virtuoso con la formazione: Mohr dimostra che i cambiamenti della collocazione individuale possono indirizzare le decisioni d'investimento dei datori di lavoro in direzione di un maggior coinvolgimento nella formazione anche del personale scarsamente qualificato (Mohr et al. 2016).

Tutte le organizzazioni si stanno riposizionando nei nuovi scenari della competitività soprattutto attraverso il potenziamento e l'utilizzo quasi esclusivo di personale qualificato e sempre più spesso la chiave di volta viene indentificata con una formazione di qualità. Una recente indagine (Kashif et al. 2020) individuando una stretta relazione tra le pratiche formative e la performance dei dipendenti e le relative ricadute organizzative, sottolinea alcuni elementi del processo da cui dipende concretamente qualità ed efficacia, e cioè: una buona progettazione, strettamente legata ai fabbisogni, la scelta oculata delle modalità di erogazione, con una preferenza per la formazione on the job.

Marwan e Rokaya considerano il processo di formazione una "variabile moderatrice" che ha un ruolo significativo nel migliorare il rapporto tra l'idoneità al lavoro del dipendente e il suo coinvolgimento organizzativo (Marwan e Rokaya 2020). Altri studi hanno mostrato, utilizzando il metodo di regressione lineare multipla, che la formazione professionale (insieme con la retribuzione) ha una influenza significativa sull'efficacia del lavoro dei dipendenti nel comparto delle assicurazioni (Abadi e Perkasa 2020).

Lukowski, basandosi sui dati aziendali del BIBB Training Panel, applica un modello logit per considerare il ruolo svolto dall'uso della tecnologia nella partecipazione dei lavoratori alla formazione (Lukowski et al. 2020). I risultati suggeriscono che le aziende con una percentuale maggiore di dipendenti 
coinvolti in attività connesse all'uso delle tecnologie digitali avviano un maggior numero di lavoratori in formazione ma, complessivamente, per un volume di ore pro/capite minore rispetto ad altri settori. Gli autori suggeriscono una possibile spiegazione nel fatto che dopo la formazione iniziale sull'uso delle tecnologie digitali prendono avvio effetti di apprendimento cumulativi e sostanziali attraverso l'esperienza e la pratica diretta e che rendono quest'ultima più efficace di ogni altro intervento.

Molto interessanti sono infine gli esiti di una recente indagine di Damei che evidenziano una relazione significativa e positiva tra formazione e fidelizzazione dei dipendenti, nonché tra quest'ultima e le pratiche di rotazione del lavoro, di coaching e di mentoring: il vantaggio competitivo aziendale, dunque, si gioca in questi casi sulla valorizzazione esplicita del capitale umano (Damei 2020).

\section{L'accrescimento della conoscenza attraverso la formazione sulle 'tecnologie abilitanti' nella percezione dei lavoratori coinvolti}

A partire da precedenti studi e classificazioni ed in particolare dall'ormai classico lavoro realizzato da Boston Consulting che poneva alla base della cosiddetta quarta rivoluzione industriale nove cluster tecnologici definiti 'abilitanti', I'Osservatorio Industria 4.0 del Politecnico di Milano ha definito sei diversi 'insiemi' di tecnologie (o tecnologie digitali innovative) suddivise in due 'sottoinsiemi'. II primo raggruppa le tecnologie dell'informazione (IT) ed in particolare: Industrial Internet of Things, Industrial Analytics (Big Data) e Cloud Manufacturing. II secondo raggruppa le tecnologie operazionali (OT) che sono nel dettaglio: Advanced Automation (robotica collaborativa), Advanced Human Machine Interface (HMI) e Additive Manufacturing.

Tabella 1. Le sei 'tecnologie abilitanti'

Internet of Things: oggetti fisici intelligenti che comunicano tra loro e con le persone attraverso Internet. Questi oggetti forniscono informazioni quali lo "stato di funzionamento", la "localizzazione" e la "tracciabilità"

Industrial analytics (Big Data e Big Data Analytics): applicazione di nuove tecniche e strumenti di business intelligence, visualizzazione, simulazione, forecasting, data analytics per porre in evidenza l'informazione contenuta nei dati e utilizzarla per prendere decisioni

Cloud Manufacturing: tramite Internet si rende disponibile l'accesso diffuso, agevole e a richiesta di risorse virtuali, condivise e configurabili a supporto dei processi produttivi e di gestione della supply chain (catena di distribuzione)

Advanced Automation (robotica collaborativa): robot che collaborano con operatori umani nell'esecuzione dei processi produttivi

Advanced Human Machine Interface (Interfaccia uomo - macchina): dispositivi indossabili e interfacce uomo macchina intuitive per l'acquisizione e/o veicolazione di informazioni in formato vocale, visuale e tattile

Additive Manufacturing: stampa 3D, creazione oggetto, strato per strato, attraverso la stampa

Nel corso dell'indagine ROLA è stato chiesto a tutti i lavoratori partecipanti di definire su una scala di 1 a 5 il loro livello di conoscenza rispetto a ciascuna delle sei 'tecnologie abilitanti'. Particolare 
attenzione è stata posta alla comparazione tra le risposte dei lavoratori coinvolti in iniziative formative aventi ad oggetto "le innovazioni tecnologiche" e quelle dei lavoratori formati su altre tematiche ${ }^{2}$. Ai formati sulle innovazioni tecnologiche è stato chiesto, inoltre, di esprimere il livello di conoscenza (con le medesime modalità e sui medesmi sei argomenti) prima e dopo la formazione ${ }^{3}$.

Si è inteso in tal modo acquisire una prima stima del grado di conoscenza delle principali traiettorie dell'innovazione tra i lavoratori delle imprese aderenti a Fondimpresa coinvolti in formazione (che, per quanto imperfetta, è anche una indicazione del fabbisogno) e, allo stesso tempo, ottenere una misurazione (per quanto anch'essa indiretta) della diffusione di tematiche connesse alle 'tecnologie abilitanti' nelle iniziative finanziate.

Nella seguente figura 1 (dove la scala originaria 1-5 è stata convertita in 1-100) è riportato il livello medio di conoscenza delle 'tecnologie abilitanti' calcolato sulle percezioni rilevate nei due gruppi ( $i$ formati sulle innovazioni tecnologiche e i formati su altre tematiche).

Figura 1. Livello medio di conoscenza sulle 'tecnologie abilitanti' dei lavoratori coinvolti in iniziative formative aventi ad oggetto "le innovazioni tecnologiche" e dei formati su altri ambiti tematici (punteggio espresso dai partecipanti su una scala da 1 a 5 , qui convertita in scala da 1 a 100)

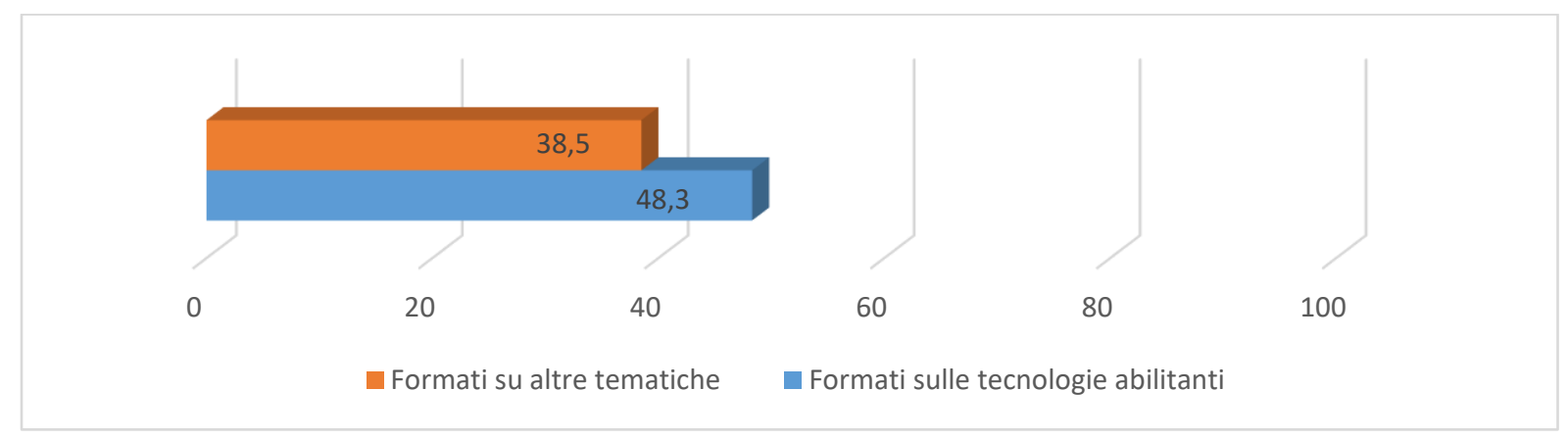

Fonte: nostre elaborazioni su dati Indagine ROLA 2019, Fondimpresa

I livelli non sono alti e la differenza tra i due aggregati non è ampia come si potrebbe supporre (circa 10 punti). In particolare, il livello medio di conoscenza percepito dai lavoratori formati su tematiche non connesse all'innovazione non è di molto inferiore all'altro e dimostra come i lavoratori delle imprese aderenti a Fondimpresa, rappresentino, comunque, un segmento della manodopera del Paese dove la tecnologia, anche nelle sue espressioni più progressive, ha già fatto il suo ingresso. II

\footnotetext{
2 I lavoratori del campione hanno indicato la tematica oggetto del corso frequentato scegliendo tra le 15 seguenti modalità: Lingue straniere; Italiano per stranieri; Vendita, marketing e comunicazione; Contabilità e finanza; Gestione aziendale e amministrazione; Lavoro d'ufficio e di segreteria; Sviluppo delle abilità personali e conoscenza del contesto lavorativo; Sviluppo di capacità manageriali; Informatica; Innovazioni tecnologiche; Innovazioni organizzative; Tecniche e tecnologie di produzione; Sicurezza sul lavoro, salvaguardia della salute, ambiente; Servizi alle persone, servizi di trasporto, vigilanza, servizi antincendio; Altro.

${ }^{3}$ I lavoratori hanno espresso il livello di conoscenza da essi percepito esprimendo un punteggio da 1 a 5, su ogni 'tecnologia abilitante', utilizzando le seguenti definizioni:

1. Nessuna conoscenza

2. La conosco poco

3. La conosco a sufficienza

4. La conosco ad un livello buono

5. La conosco perfettamente.
} 
livello medio dichiarato dai formati sull'innovazione, che si mantiene ben al di sotto della metà della scala e che non si distacca in modo significativo, potrebbe indicare invece la necessità di una maggiore specializzazione di un' offerta formativa che non incide in modo decisivo e che, sui segmenti tecnologici più avanzati, potrebbe conseguire miglioramenti ancora molto rilevanti.

Maggiori dettagli possono essere tratti dall'osservazione dei livelli di conoscenza percepiti per ciascuna delle 'tecnologie abilitanti' in tre diversi ambiti territoriali (Nord-Centro-Sud).

I valori riportati nella seguente tabella 2 evidenziano con una certa nettezza una sostanziale ininfluenza della collocazione territoriale nel determinare i livelli di conoscenza sulle 'tecnologie abilitanti' tra i lavoratori formati in ambiti non connessi alle innovazioni tecnologiche ${ }^{4}$ : i valori riscontrati nelle tre circoscrizioni territoriali, per ciascun cluster tecnologico, sono sempre compresi in un intervallo minimo di due o tre punti.

Tabella 2. Livello medio di conoscenza delle 'tecnologie abilitanti' percepito dai lavoratori formati sulle 'innovazioni tecnologiche' nel confronto con i lavoratori formati su altri ambiti tematici (punteggio espresso dai partecipanti su una scala da 1 a 5 , qui trasformato in scala da 1 a 100)

\begin{tabular}{|c|c|c|c|}
\hline & $\begin{array}{l}\text { Livello di conoscenza } \\
\text { percepito dai formati sulle } \\
\text { innovazioni tecnologiche }\end{array}$ & $\begin{array}{c}\text { Livello di conoscenza } \\
\text { percepito dai formati su } \\
\text { altri ambiti }\end{array}$ & Differenza \\
\hline \multicolumn{4}{|c|}{ Internet of Things } \\
\hline Nord & 56,9 & 42,9 & 14,1 \\
\hline Centro & 57,2 & 45,7 & 11,5 \\
\hline Mezzogiorno & 50,0 & 43,3 & 6,8 \\
\hline \multicolumn{4}{|c|}{ Industrial Analytics (Big Data) } \\
\hline Nord & 51,6 & 35,7 & 15,9 \\
\hline Centro & 50,9 & 39,5 & 11,4 \\
\hline Mezzogiorno & 45,1 & 37,7 & 7,4 \\
\hline \multicolumn{4}{|c|}{ Cloud Manufacturing } \\
\hline Nord & 54,1 & 40,3 & 13,8 \\
\hline Centro & 54,9 & 41,7 & 13,2 \\
\hline Mezzogiorno & 47,5 & 41,3 & 6,2 \\
\hline \multicolumn{4}{|c|}{ Advanced Automation } \\
\hline Nord & 46,4 & 35,9 & 10,5 \\
\hline Centro & 46,0 & 36,3 & 9,7 \\
\hline Mezzogiorno & 40,8 & 37,2 & 3,6 \\
\hline \multicolumn{4}{|c|}{ Advanced Human Machine Interface (HMI) } \\
\hline Nord & 49,3 & 38,3 & 11,1 \\
\hline Centro & 50,0 & 40,3 & 9,6 \\
\hline Mezzogiorno & 41,9 & 39,6 & 2,4 \\
\hline \multicolumn{4}{|c|}{ Additive Manufacturing } \\
\hline Nord & 44,3 & 34,4 & 9,9 \\
\hline Centro & 45,1 & 36,2 & 8,8 \\
\hline Mezzogiorno & 38,5 & 36,2 & 2,3 \\
\hline
\end{tabular}

Fonte: nostre elaborazioni su dati Indagine ROLA 2019, Fondimpresa

${ }^{4}$ I differenti valori che si osservano nei livelli percepiti di conoscenza tra i diversi ambiti tecnologici (la conoscenza dell'Internet of Things sembra essere generalmente più diffusa rispetto all'Additive Manufacturing indipendentemente dal territorio e dal coinvolgimento in formazione di diverso tema) non sono oggetto specifico di attenzione di questi studio e sono probabilmente attribuibili alla loro differente diffusione (quest'ultima, peraltro, non quantificabile con la strumentazione qui utilizzata). 
Ciò che fa la differenza è invece la partecipazione alla formazione: i formati sulle innovazioni tecnologiche del Nord e del Centro (e, comunque, in misura maggiore sempre quelli del Nord) dichiarano infatti livelli di conoscenza sistematicamente maggiori dei lavoratori formati in altri ambiti rispetto a quanto accade ai lavoratori del Sud, dove la differenza tra i due gruppi è molto più sfumata. Ciò si riscontra con riferimento a ciascuna delle sei 'tecnologie abilitanti': i livelli di conoscenza rilevati tra i lavoratori del Sud variano da un minimo di 2,3 punti per l'Additive Manufacturing ad un massimo di 7,4 per l'Industrial Analytics. Regolarmente più alti sono i valori registrati al Nord e al Centro che, per i medesimi due cluster, sono pari rispettivamente a 9,9 e 15,9 punti.

Risultati solo apparentemente contraddittori possono essere tratti dai dati della seguente tabella 3, dove si riportano, sempre per territorio, i livelli di conoscenza percepiti, riguardo le 'tecnologie abilitanti', considerando solo i lavoratori coinvolti in iniziative formative sulle 'innovazioni tecnologiche', prima e dopo la formazione.

Tabella 3. Livello medio di conoscenza delle 'tecnologie abilitanti' percepito dai lavoratori formati sulle 'innovazioni tecnologiche' prima e dopo la formazione (punteggio espresso dai partecipanti su una scala da 1 a 5 , qui trasformato in scala da 1 a 100)

\begin{tabular}{lccc}
\hline & $\begin{array}{c}\text { Livello di conoscenza } \\
\text { percepito prima della } \\
\text { formazione }\end{array}$ & $\begin{array}{c}\text { Livello di conoscenza } \\
\text { percepito dopo la } \\
\text { formazione }\end{array}$ & Differenza \\
\hline Internet of Things & & & $\mathbf{4 , 8}$ \\
Nord & 52,1 & 56,9 & $\mathbf{7 , 5}$ \\
Centro & 49,7 & 57,2 & $\mathbf{9 , 0}$ \\
Mezzogiorno & 41,0 & 50,0 & $\mathbf{9 , 1}$ \\
\hline Industrial Analytics (Big Data) & & & $\mathbf{8 , 1}$ \\
Nord & 42,5 & 51,6 & $\mathbf{8 , 3}$ \\
Centro & 42,8 & 50,9 & $\mathbf{7 , 2}$ \\
Mezzogiorno & 36,8 & 45,1 & $\mathbf{9 , 6}$ \\
\hline Cloud Manufacturing & & & $\mathbf{8 , 3}$ \\
Nord & 46,9 & 54,1 & $\mathbf{4 , 5}$ \\
Centro & 45,3 & 54,9 & $\mathbf{6 , 2}$ \\
Mezzogiorno & 39,2 & 47,5 & $\mathbf{6 , 6}$ \\
\hline Advanced Automation & & 46,4 & $\mathbf{6 , 3}$ \\
Nord & 41,9 & 46,0 & $\mathbf{7 , 0}$ \\
Centro & 39,8 & 40,8 & $\mathbf{6 , 3}$ \\
Mezzogiorno & 34,2 & & $\mathbf{5 , 6}$ \\
\hline Advanced Human Machine Interface (HMI) & & 49,3 & $\mathbf{5 , 5}$ \\
Nord & 43,0 & 50,0 & $\mathbf{6 , 1}$ \\
Centro & 43,0 & 41,9 & \\
Mezzogiorno & 35,6 & 44,3 & 45,1 \\
\hline Additive Manufacturing & & 38,5 & \\
Nord & 38,7 & & \\
Menzogiorno & 39,6 & 32,4 & \\
\hline
\end{tabular}

Fonte: nostre elaborazioni su dati Indagine ROLA 2019, Fondimpresa

Qui si osserva, infatti, una differenza tra le diverse aree territoriali molto meno marcata e, nella maggioranza dei casi, opposta alla precedente: I'accrescimento della conoscenza percepita dopo la formazione è maggiore tra i lavoratori del Sud rispetto a quelli del Nord per la maggior parte dei cluster tecnologici considerati. 
A ben vedere ciò è perfettamente coerente con quanto si osserva in genere nelle analisi di impatto della formazione. Per quanto riguarda le differenze tra la percezione dei lavoratori formati sulle 'innovazioni tecnologiche' e quelli formati su altri temi (i dati in tabella 2), gioca senz'altro un ruolo decisivo il legame e l'inerenza tra le tematiche formative concretamente trattare nei corsi e le 'tecnologie abilitanti' qui considerate: evidentemente la formazione sull'innovazione, promossa da Fondimpresa al Nord e al Centro nel periodo di riferimento dell'indagine, è stata maggiormente indirizzata sui tracciati tecnologici più avanzati rispetto a quanto accaduto nel Mezzogiorno. Ovviamente potrebbe sorgere l'esigenza di chiarire quanto ciò dipenda dalle caratteristiche dei sistemi formativi locali (e quindi dall'offerta di formazione) e quanto invece dalle peculiarità e dal diverso posizionamento nei confronti dell'innovazione del tessuto produttivo dei diversi territori (e quindi dalle caratteristiche della domanda). I dati a disposizione non consentono valide inferenze di questo tipo; tuttavia, sembra molto probabile una compresenza di entrambi i fattori, che sono del resto, quasi sempre, fortemente e strutturalmente connessi.

Per quanto riguarda invece la maggiore differenza riscontrata nei livelli di conoscenza percepiti dai formati sulle 'innovazioni tecnologiche' del Sud dopo la formazione rispetto ai lavoratori del Nord ( $\mathrm{i}$ dati in tabella 3), è molto probabile che, in un tessuto produttivo relativamente meno avanzato, nel quale i lavoratori non sono stati ancora adeguatamente coinvolti nell'uso delle tecnologie maggiormente innovative, una formazione ben progettata (anche se non eccessivamente specializzata) abbia avuto un rendimento marginale più elevato, soprattutto nella percezione del partecipante.

Ciò, insieme alle ovvie esigenze equitative e come rilevato più volte negli studi in materia, rafforza le ragioni che inducono alla realizzazione di maggiori e più rilevanti investimenti formativi rivolti ai lavoratori delle imprese del Sud.

Un passo ulteriore per mettere a fuoco ciò che maggiormente ha condizionato le percezioni relative al grado di conoscenza sulle 'tecnologie abilitanti', può essere compiuto utilizzando la seguente equazione di regressione:

$$
y=\beta_{0}+\beta_{1} * D+\beta_{2} * A+\beta_{3} * C+\varepsilon
$$

dove l'outcome (y) rappresenta il livello di conoscenza percepito relativamente alle sei tecnologie abilitanti' (il punteggio è espresso in logaritmo) e i gruppi di variabili esplicative considerati riguardano: - (D) le caratteristiche del lavoratore: genere, età, titolo di studio, inquadramento, tipologia contrattuale, area di attività, mansioni; - (A) le caratteristiche dell'impresa di appartenenza: dimensione e settore di attività; - $(C)$ le caratteristiche della formazione fruita: durata, canale di finanziamento, presenza di metodologie diverse dall'aula, coerenza dell'iniziativa con le mansioni svolte, coinvolgimento del responsabile aziendale su obiettivi e risultati dell'iniziativa formativa.

La seguente tabella 4 evidenzia in primo luogo l'effetto sui livelli di conoscenza delle iniziative formative centrate sulle 'innovazioni tecnologiche' rispetto a quelle dedicate ad altri temi. Sul punto, si conferma quanto già osservato in precedenza: al Nord e al Centro l'impatto è maggiore. I formati sulle innovazioni dichiarano livelli di conoscenza più alti di circa di circa il 20\% (0.1951 al Nord e 0.2002 
al Centro ${ }^{5}$ ); più contenuto il vantaggio registrato al Sud, pari al 7,8\% (0.078), e comunque, anche in questo caso, ciò è coerente con il delta meno ampio evidenziato nel Mezzogiorno nella precedente analisi sui dati in tabella 2.

Tabella 4. Livelli di conoscenza sulle 'tecnologie abilitanti' percepiti dai lavoratori partecipanti all'indagine ROLA in relazione alle caratteristiche dei lavoratori formati, delle imprese di appartenenza e della formazione fruita

\begin{tabular}{|c|c|c|c|}
\hline Variabili esplicative & Nord & Centro & Sud \\
\hline $\begin{array}{l}\text { Formati sulle 'innovazioni tecnologiche' nel confronto con i formati sulle altre } \\
\text { tematiche }\end{array}$ & $\begin{array}{c}0.1951^{* * *} \\
{[0.0513]}\end{array}$ & $\begin{array}{c}0.2002^{* * *} \\
{[0.0434]}\end{array}$ & $\begin{array}{l}0.0781^{*} \\
{[0.0414]}\end{array}$ \\
\hline \multicolumn{4}{|l|}{ Classi d'età } \\
\hline Da 30 a 49 anni nel confronto con la classe 'fino a 29 anni' & $\begin{array}{l}-0.0476^{*} \\
{[0.0258]}\end{array}$ & $\begin{array}{l}-0.1105^{* *} \\
{[0.0497]}\end{array}$ & $\begin{array}{l}-0.0544 \\
{[0.0353]}\end{array}$ \\
\hline Oltre 50 anni nel confronto con la classe 'fino a 29 anni' & $\begin{array}{l}-0.1353^{* * *} \\
{[0.0295]}\end{array}$ & $\begin{array}{l}-0.1857^{* * *} \\
{[0.0528]}\end{array}$ & $\begin{array}{c}-0.0349 \\
{[0.0418]}\end{array}$ \\
\hline \multicolumn{4}{|l|}{ Titolo di studio } \\
\hline Diploma nel confronto con la Licenza Media & $\begin{array}{c}0.0571^{* *} \\
{[0.0287]}\end{array}$ & $\begin{array}{l}0.0734^{*} \\
{[0.0435]}\end{array}$ & $\begin{array}{l}0.0778^{* *} \\
{[0.0331]}\end{array}$ \\
\hline Laurea e post-laurea nel confronto con la Licenza Media & $\begin{array}{c}0.0572 \\
{[0.0377]}\end{array}$ & $\begin{array}{c}0.1046^{* *} \\
{[0.0515]}\end{array}$ & $\begin{array}{c}0.1396 * * * \\
{[0.0476]}\end{array}$ \\
\hline Lavoratrici nel confronto con i lavoratori & $\begin{array}{l}-0.1093^{* * *} \\
{[0.0256]}\end{array}$ & $\begin{array}{l}-0.1418 * * * \\
{[0.0283]}\end{array}$ & $\begin{array}{l}-0.0052 \\
{[0.0316]}\end{array}$ \\
\hline \multicolumn{4}{|l|}{ Inquadramento } \\
\hline Impiegati nel confronto con gli operai & $\begin{array}{c}0.1494 * * * \\
{[0.0340]}\end{array}$ & $\begin{array}{c}0.0472 \\
{[0.0457]}\end{array}$ & $\begin{array}{c}0.0454 \\
{[0.0427]}\end{array}$ \\
\hline Quadri nel confronto con gli operai & $\begin{array}{c}0.2118^{* * *} \\
{[0.0418]}\end{array}$ & $\begin{array}{c}0.1128^{* *} \\
{[0.0569]}\end{array}$ & $\begin{array}{c}0.2436 * * * \\
{[0.0770]}\end{array}$ \\
\hline \multicolumn{4}{|l|}{ Tipologia contrattuale } \\
\hline Tempo determinato nel confronto con il Tempo Indeterminato & $\begin{array}{c}-0.0436 \\
{[0.0393]}\end{array}$ & $\begin{array}{c}0.0302 \\
{[0.0519]}\end{array}$ & $\begin{array}{l}-0.0655 \\
{[0.0433]}\end{array}$ \\
\hline Part time nel confronto con il Tempo Indeterminato & $\begin{array}{c}0.1372 \\
{[0.0882]}\end{array}$ & $\begin{array}{c}0.0539 \\
{[0.1606]}\end{array}$ & $\begin{array}{c}0.0035 \\
{[0.0574]}\end{array}$ \\
\hline Altro contratto nel confronto con il Tempo Indeterminato & $\begin{array}{c}0.2808^{* *} \\
{[0.1126]}\end{array}$ & $\begin{array}{c}0.0604 \\
{[0.1325]}\end{array}$ & $\begin{array}{c}-0.2537^{* *} \\
{[0.1231]}\end{array}$ \\
\hline \multicolumn{4}{|l|}{ Area di attività } \\
\hline Commerciale/marketing & $\begin{array}{c}-0.0186 \\
{[0.0501]}\end{array}$ & $\begin{array}{c}0.0426 \\
{[0.0503]}\end{array}$ & $\begin{array}{c}-0.0925 \\
{[0.0568]}\end{array}$ \\
\hline Logistica/magazzino & $\begin{array}{c}-0.0463 \\
{[0.0464]}\end{array}$ & $\begin{array}{c}-0.0987^{*} \\
{[0.0579]}\end{array}$ & $\begin{array}{l}-0.0229 \\
{[0.0659]}\end{array}$ \\
\hline Produzione & $\begin{array}{c}0.0531 \\
{[0.0378]}\end{array}$ & $\begin{array}{c}0.0007 \\
{[0.0424]}\end{array}$ & $\begin{array}{l}-0.0533 \\
{[0.0493]}\end{array}$ \\
\hline Ricerca e sviluppo & $\begin{array}{c}0.1230 * * * \\
{[0.0438]}\end{array}$ & $\begin{array}{c}-0.0332 \\
{[0.0632]}\end{array}$ & $\begin{array}{c}0.0295 \\
{[0.0840]}\end{array}$ \\
\hline Supporto tecnico/manutenzione & $\begin{array}{l}0.0790 * \\
{[0.0405]}\end{array}$ & $\begin{array}{c}0.1099 * * \\
{[0.0499]}\end{array}$ & $\begin{array}{c}0.0255 \\
{[0.0626]}\end{array}$ \\
\hline Vendita & $\begin{array}{c}0.0371 \\
{[0.0563]}\end{array}$ & $\begin{array}{l}-0.0419 \\
{[0.0690]}\end{array}$ & $\begin{array}{c}-0.1278 \\
{[0.0848]}\end{array}$ \\
\hline
\end{tabular}

\footnotetext{
${ }^{5}$ Per una maggiore chiarezza espositiva i dati della tabella sono discussi nel testo moltiplicati per 100. La stessa modalità è adottata per le elaborazioni successive.
} 


\begin{tabular}{|c|c|c|c|}
\hline Variabili esplicative & Nord & Centro & Sud \\
\hline \multicolumn{4}{|c|}{ Mansioni svolte (nel confronto con la mansione: "Pianificazione e coordinamento delle strategie aziendali") } \\
\hline Analisi di situazioni complesse, sviluppo di nuove conoscenze, diagnosi e ricerca & $\begin{array}{c}0.0496 \\
{[0.0433]}\end{array}$ & $\begin{array}{c}0.0037 \\
{[0.0538]}\end{array}$ & $\begin{array}{c}0.0253 \\
{[0.0765]}\end{array}$ \\
\hline Controllo dei processi di produzione e applicazioni di protocolli operativi & $\begin{array}{c}-0.007 \\
{[0.0439]}\end{array}$ & $\begin{array}{l}-0.0518 \\
{[0.0503]}\end{array}$ & $\begin{array}{c}0.0124 \\
{[0.0576]}\end{array}$ \\
\hline Acquisizione, trattamento, archiviazione e trasmissione delle informazioni & $\begin{array}{c}-0.0979 * * \\
{[0.0447]}\end{array}$ & $\begin{array}{c}-0.1449 * * * \\
{[0.0496]}\end{array}$ & $\begin{array}{c}-0.028 \\
{[0.0565]}\end{array}$ \\
\hline Assistenza ai clienti, ai consumatori, ai cittadini & $\begin{array}{l}-0.1276^{* * *} \\
{[0.0473]}\end{array}$ & $\begin{array}{l}-0.0347 \\
{[0.0537]}\end{array}$ & $\begin{array}{l}-0.0628 \\
{[0.0525]}\end{array}$ \\
\hline Costruzione, riparazione o manutenzione di manufatti, oggetti e macchine & $\begin{array}{l}-0.0345 \\
{[0.0516]}\end{array}$ & $\begin{array}{c}-0.1441^{* *} \\
{[0.0606]}\end{array}$ & $\begin{array}{l}-0.1153 \\
{[0.0790]}\end{array}$ \\
\hline $\begin{array}{l}\text { Conduzione e controllo di macchine industriali e di impianti automatizzati o } \\
\text { robotizzati }\end{array}$ & $\begin{array}{l}-0.0242 \\
{[0.0565]}\end{array}$ & $\begin{array}{l}-0.0573 \\
{[0.0664]}\end{array}$ & $\begin{array}{c}0.0624 \\
{[0.0592]}\end{array}$ \\
\hline Compiti di manovalanza in agricoltura edilizia e industria & $\begin{array}{l}-0.1833^{* * *} \\
{[0.0580]}\end{array}$ & $\begin{array}{c}-0.1572 * * \\
{[0.0747]}\end{array}$ & $\begin{array}{c}-0.1308^{* *} \\
{[0.0627]}\end{array}$ \\
\hline \multicolumn{4}{|c|}{ Settore di attività dell'impresa (nel confronto con il settore "Servizi alle imprese") } \\
\hline Industria metalmeccanica & $\begin{array}{c}0.0206 \\
{[0.0312]}\end{array}$ & $\begin{array}{c}-0.0251 \\
{[0.0385]}\end{array}$ & $\begin{array}{c}0.1737^{* * *} \\
{[0.0435]}\end{array}$ \\
\hline Altra manifattura & $\begin{array}{c}0.0223 \\
{[0.0327]}\end{array}$ & $\begin{array}{c}0.0281 \\
{[0.0371]}\end{array}$ & $\begin{array}{c}0.0459 \\
{[0.0385]}\end{array}$ \\
\hline Costruzioni e altre industrie non manifatturiera & $\begin{array}{c}-0.1070^{*} \\
{[0.0577]}\end{array}$ & $\begin{array}{c}-0.1240 * * \\
{[0.0546]}\end{array}$ & $\begin{array}{l}0.1246^{*} \\
{[0.0645]}\end{array}$ \\
\hline Commercio, alberghi e ristoranti & $\begin{array}{l}-0.0231 \\
{[0.0407]}\end{array}$ & $\begin{array}{c}-0.0789 * \\
{[0.0462]}\end{array}$ & $\begin{array}{c}0.0581 \\
{[0.0500]}\end{array}$ \\
\hline Sanità, assistenza e servizi alle persone & $\begin{array}{c}-0.0994^{* *} \\
{[0.0496]}\end{array}$ & $\begin{array}{c}-0.1025^{*} \\
{[0.0541]}\end{array}$ & $\begin{array}{c}0.1107^{* * *} \\
{[0.0413]}\end{array}$ \\
\hline \multicolumn{4}{|l|}{ Classi dimensionali dell'impresa } \\
\hline Da 50 a 249 dipendenti nel confronto con le imprese con meno di 50 & $\begin{array}{c}-0.0454 \\
{[0.0296]}\end{array}$ & $\begin{array}{c}-0.0103 \\
{[0.0296]}\end{array}$ & $\begin{array}{c}-0.1166^{* * *} \\
{[0.0301]}\end{array}$ \\
\hline Oltre 250 dipendenti nel confronto con le imprese con meno di 50 & $\begin{array}{l}-0.0349 \\
{[0.0326]}\end{array}$ & $\begin{array}{l}0.0654^{*} \\
{[0.0370]}\end{array}$ & $\begin{array}{c}0.0008 \\
{[0.0418]}\end{array}$ \\
\hline Durata della formazione & $\begin{array}{c}0.0015^{* * *} \\
{[0.0005]}\end{array}$ & $\begin{array}{c}-0.0009 \\
{[0.0006]}\end{array}$ & $\begin{array}{c}0.0007 \\
{[0.0005]}\end{array}$ \\
\hline \multicolumn{4}{|c|}{ Condivisione dell'esperienza formativa con il responsabile (nel confronto con la completa assenza di condivisione) } \\
\hline Prima della formazione & $\begin{array}{c}0.0567 \\
{[0.0356]}\end{array}$ & $\begin{array}{l}0.0870^{*} \\
{[0.0458]}\end{array}$ & $\begin{array}{l}0.1442^{* *} \\
{[0.0638]}\end{array}$ \\
\hline Dopo la formazione & $\begin{array}{c}0.0805^{* *} \\
{[0.0314]}\end{array}$ & $\begin{array}{c}0.1038^{* *} \\
{[0.0412]}\end{array}$ & $\begin{array}{l}0.1387^{* *} \\
{[0.0578]}\end{array}$ \\
\hline Sia prima che dopo & $\begin{array}{c}0.1342^{* * *} \\
{[0.0301]}\end{array}$ & $\begin{array}{c}0.1538^{* * *} \\
{[0.0352]}\end{array}$ & $\begin{array}{c}0.1382^{* * *} \\
{[0.0478]}\end{array}$ \\
\hline $\begin{array}{l}\text { Finanziamento dell'iniziativa con il "Conto Formazione" nel confronto con il } \\
\text { "Conto di Sistema" }\end{array}$ & $\begin{array}{c}0.0364 \\
{[0.0255]}\end{array}$ & $\begin{array}{c}-0.1487^{* * *} \\
{[0.0305]}\end{array}$ & $\begin{array}{l}-0.1009 * * * \\
{[0.0322]}\end{array}$ \\
\hline $\begin{array}{l}\text { Iniziative formative maggiormente coerenti alle mansioni svolte in impresa } \\
\text { (nella percezione del lavoratore) }\end{array}$ & $\begin{array}{c}0.0582 \\
{[0.0493]}\end{array}$ & $\begin{array}{c}0.0018 \\
{[0.0520]}\end{array}$ & $\begin{array}{c}0.0372 \\
{[0.0590]}\end{array}$ \\
\hline Formazione realizzata anche con metodologie diverse dall'aula & $\begin{array}{c}0.0353 \\
{[0.0338]}\end{array}$ & $\begin{array}{l}-0.0143 \\
{[0.0358]}\end{array}$ & $\begin{array}{l}0.1206^{* *} \\
{[0.0480]}\end{array}$ \\
\hline Costante & $\begin{array}{c}0.3552^{* * *} \\
{[0.0832]}\end{array}$ & $\begin{array}{c}0.7833^{* * *} \\
{[0.1160]}\end{array}$ & $\begin{array}{c}0.3508^{* * *} \\
{[0.1224]}\end{array}$ \\
\hline Totale osservazioni $(N)$ & 2452 & 1436 & 1447 \\
\hline$R^{2}$ & 0.2264 & 0.2032 & 0.1626 \\
\hline
\end{tabular}

Nota: i valori esprimono il logaritmo del punteggio medio dichiarato dai lavoratori per i sei diversi cluster tecnologici. Assumono valore positivo o negativo a seconda del segno della relazione con la variabile esplicativa (diretta o inversa). Gli asterischi indicano tre diversi livelli di significatività: ${ }^{* * *} p<0.01,{ }^{* *} p<0.05, * p<0.1$.

Fonte: nostre elaborazioni su dati Indagine ROLA 2019, Fondimpresa 
Risultano significativi gli effetti dell'età: come si poteva presumere i lavoratori più giovani sembrano maggiormente ricettivi, infatti i livelli di conoscenza diminuiscono ovunque al crescere dell'età, sebbene in modo più marcato al Centro rispetto al Nord e al Sud. Si rileva un'influenza abbastanza marcata anche del titolo di studio e ancor più dell'inquadramento aziendale: al crescere della scolarizzazione cresce la 'conoscenza tecnologica', soprattutto tra i laureati del Sud ed anche del Centro; molto evidente è poi la differenza tra i quadri e gli operai (e molto minore invece la differenza tra questi ultimi e gli impiegati).

La diffusione della conoscenza sulle tecnologie di punta è quindi maggiormente accentuata tra $i$ lavoratori più giovani, con titolo di studio elevati e con un posizionamento di maggior responsabilità nella gerarchia aziendale. E tra questi, i maggiori livelli si registrano tra i lavoratori del Mezzogiorno. Di particolare interesse l'effetto di segno opposto che si produce al Nord rispetto al Sud per quanto riguarda le diverse tipologie contrattuali: al Nord, tra i lavoratori con contratti 'atipici' (diversi sia dal tempo indeterminato, sia dal tempo determinato, anche part-time) si registrano livelli di conoscenza maggiori rispetto a quelli con forme contrattuali più stabili, al contrario di quanto avviene al Sud. Evidentemente (come più volte osservato in letteratura), la flessibilità svolge un ruolo molto differente nelle due aree del Paese: al Nord viene adottata anche per funzioni e figure inserite negli snodi tecnologicamente rilevanti dei processi produttivi, al Sud è ancora maggiormente destinata a compiti e mansioni di contenuto più elementare.

La relazione inversa tra i livelli di conoscenza tecnologica e complessità delle mansioni svolte è del resto ben illustrata dalle altre evidenze, in particolare per le attività più elementari come i compiti di manovalanza in agricoltura, manifattura ed edilizia, ma anche per la riparazione e manutenzione oppure per l'acquisizione, trattamento e trasmissione delle informazioni dove si collocano molte delle normali funzioni di assistenza e segreteria d'ufficio e nelle quali l'utilizzo delle tecnologie informatiche, pur diffuso, è limitato spesso al semplice "livello utente".

Relazioni di segno analogo si osservano del resto anche per quanto riguarda il settore di attività dell'impresa di appartenenza ed in particolare nel confronto tra un ambito di attività peculiare e caratteristico come i servizi alle imprese, nel quale si collocano sovente le realtà avanzate non solo del terziario, e i settori tradizionali delle costruzioni, dei servizi, e dell'assistenza, dove invece la diffusione delle nuove tecnologie è strutturalmente inferiore. Tra l'altro, ciò concorre a chiarire anche lo svantaggio di genere che si osserva in particolare al Centro e al Nord, con livelli inferiori rispettivamente del $14 \%$ e dell' $11 \%$ (0.1418 e 0.1093), dovuto essenzialmente alla differente composizione di genere nella forza lavoro nei diversi comparti così come riflessa concretamente nel campione. Quasi indifferente, invece, l'appartenenza di genere al Sud, dove però la partecipazione femminile al mercato del lavoro è più limitata e dove i maggiori ostacoli all'inserimento possono selezionare di fatto una quota maggiore di lavoratrici, spesso altamente scolarizzate, che si inseriscono efficacemente anche nei settori e nei processi produttivi comparativamente più avanzati Probabile oggetto di una messa a fuoco più precisa in occasione delle prossime edizioni dell'indagine ROLA sarà il decrescere dei livelli di conoscenza tecnologica all'aumentare della dimensione di impresa, osservabile soprattutto al Sud, dove si registra il $12 \%$ in meno (0.1166) tra i lavoratori delle medie imprese (50-249 dipendenti) nel confronto con quelli delle piccole (meno di 50 dipendenti). Tra le variabili esplicative considerate, quelle relative alle caratteristiche delle iniziative formative hanno forse un ruolo ancora più importante di altre nell'accrescimento della conoscenza tecnologica 
dei lavoratori coinvolti: emerge, ad esempio, la "condivisione del percorso formativo con il responsabile aziendale", che rappresenta, nei suoi diversi aspetti e modalità, una misura indiretta ma attendibile del grado di coerenza e appropriatezza dell'esperienza di formazione con le reali esigenze aziendali. Ovunque la relazione è positiva e, come prevedibile, si conseguono i risultati migliori dove la condivisione è più stretta e sistematica: i lavoratori che hanno condiviso l'esperienza, sia prima, sia dopo la formazione, hanno sempre dichiarato livelli di conoscenza tecnologica più alti, soprattutto nel Mezzogiorno, anche nei casi in cui la condivisione è stata meno strutturata (solo prima o solo dopo). Molto importante si rivela, sempre al Sud, l'approccio metodologico: tra i lavoratori coinvolti in iniziative formative condotte con modalità diverse dall'aula e nelle quali sono state impiegate metodologie esperienziali, si registra il $12 \%$ in più rispetto a chi si è formato in percorsi tradizionali (0.1206).

Un cenno, infine, alle modalità di finanziamento. Ricordiamo sinteticamente che Fondimpresa finanzia la formazione delle sue aziende aderenti attraverso le due modalità del Conto aziendale e del Conto di sistema: più autonomia dell'impresa nel primo caso (dove questa può disporre della quota maggioritaria dei suoi versamenti presentando, quando vuole, un Piano formativo al Fondo per la valutazione), più mutualità, più possibilità per il Fondo di indirizzare la domanda e più opportunità per le piccole imprese nel secondo caso (dove il Fondo stesso emana periodicamente Avvisi, che possono anche riguardare temi, settori o territori specifici, a fronte dei quali le imprese presentano le loro proposte, spesso raccolte, sistematizzate e realizzate dalle strutture dell'offerta formativa del territorio). A questo riguardo, emerge con evidenza dai dati una relazione inversa tra l'uso del Conto aziendale e i livelli di conoscenza, in particolare al Centro e al Sud. In altri termini, i lavoratori coinvolti nelle iniziative realizzate in questi ambiti territoriali attraverso il Conto di sistema, quindi con gli Avvisi e con una maggiore partecipazione delle imprese minori, dichiarano livelli di conoscenza tecnologica maggiori rispetto a quelli coinvolti nella formazione finanziata con i Conti aziendali. Ciò conferma senz'altro l'efficacia degli indirizzi (sulle tematiche formative, sugli approcci metodologici, sulle tipologie di impresa, sulle filiere o sui settori) che la concertazione tra le Parti Sociali determina e che il Fondo, di volta in volta, introduce negli Avvisi.

\section{Le determinanti dei cambiamenti percepiti dai lavoratori formati relativamente alle mansioni svolte e agli assetti produttivi e organizzativi}

Di solito, nelle indagini di tipo valutativo sugli effetti della formazione vengono introdotte domande rivolte alla raccolta delle percezioni dei partecipanti circa gli effetti concretamente riscontrati ex post. Nella letteratura anglosassone spesso si rilevano anche gli effetti più propriamente connessi alla carriera e alla retribuzione. Nell'indagine ROLA, maggiormente centrata sugli aspetti relativi alla diffusione e all'acquisizione di competenze sulle nuove tecnologie, è stato chiesto ai partecipanti di esprimere le proprie percezioni sia sui cambiamenti indotti nelle mansioni svolte, sia sui cambiamenti riscontrati nella vita d'impresa (nell'organizzazione interna, nella divisione dei compiti, nelle relazioni tra le aree funzionali, ecc.). Le risposte hanno evidenziato effetti rilevanti e generalmente positivi per I'approfondimento completo dei quali si rimanda al Report di indagine (Fondimpresa et al. 2020). In 
questa sede si intende, invece, porre particolare attenzione su alcune significative differenze riscontrate tra le diverse aree territoriali del Paese relativamente alla percezione del cambiamento nell' esercizio delle mansioni individuali di lavoro ${ }^{6}$ anche in connessione con le tematiche formative e con i livelli di conoscenza sulle 'tecnologie abilitanti'.

Attraverso l'utilizzo della seguente equazione di regressione:

$$
y=\beta_{0}+\beta_{1} * \ln (\text { punt })+\beta_{2} * D+\beta_{3} * A+\beta_{4}{ }^{*} C+\varepsilon
$$

è stata misurata la probabilità che i lavoratori percepiscano dei cambiamenti a seguito della formazione fruita in relazione alle diverse variabili esplicative già considerate nel precedente paragrafo. Si considera una variabile dummy con valore 1 in caso di risposta positiva per uno qualsiasi dei cambiamenti considerati nella domanda e valore 0 nel caso non fosse percepito nessun tipo di cambiamento. Le variabili esplicative sono, come detto, le stesse considerate nell'equazione di cui al precedente paragrafo, suddivise nei medesimi tre gruppi: • (D) le caratteristiche del lavoratore; $\bullet$ (A) le caratteristiche dell'impresa di appartenenza; • (C) le caratteristiche della formazione fruita. Tra queste è stata inserita un'ulteriore variabile $\left(\beta_{1}{ }^{*} \ln (\right.$ punt $\left.)\right)$ relativa alla media dei livelli di conoscenza indicati dagli intervistati su ognuna delle sei 'tecnologie abilitanti'.

I risultati dell'elaborazione, riportati nella successiva tabella 5, evidenziano sensibili differenze tra le percezioni raccolte tra i lavoratori formati del Nord e del Centro rispetto a quelle espresse dai lavoratori del Sud. Emerge infatti con una certa evidenza, e in connessione con diverse delle variabili esplicative considerate, una diversa dinamica degli effetti della formazione per quanto riguarda la percezione di mutamenti nei ruoli, nei posizionamenti e nelle mansioni svolte nel processo di produzione. Ciò si verifica nonostante siano stati invece notati effetti uniformi tra i territori nelle percezioni relative all'accrescimento delle conoscenze individuali, come illustrato ampiamente in precedenza, anche se in misura minore al Sud rispetto al Nord e al Centro.

Come ovvio, i due aspetti possono ben essere disgiunti: all'accrescimento delle conoscenze prodotto dalla formazione non sempre corrisponde una concreta e uniforme ricaduta nell'esercizio del proprio lavoro. Tra i due aspetti possono frapporsi numerose e articolate circostanze e condizioni di contesto. L'aver svolto formazione specifica sulle 'innovazioni tecnologiche' (una delle condizioni che in precedenza avevano meglio spiegato l'accrescimento di 'conoscenza tecnologica') si associa alla percezione di cambiamenti nelle mansioni tra i lavoratori al Nord e del Centro con una probabilità maggiore rispettivamente di 11 e di 13 p.p. (0.1065 e 0.1318) rispetto ai lavoratori che hanno seguito corsi su tematiche diverse, ma ciò non avviene tra i lavoratori del Mezzogiorno.

\footnotetext{
${ }^{6}$ La domanda sulla percezione dei cambiamenti nelle mansioni individuali era la seguente:

"Se le sue mansioni sono cambiate nel periodo compreso tra la fine della formazione ad oggi, può indicare in che modo? (barrare al massimo 2 opzioni):

- $\quad$ Aumento del numero di attività da svolgere

- Maggior numero di responsabilità

- Diminuzione del numero di attività da svolgere

- Coordinamento di un gruppo di lavoro

- Avanzamento di carriera

- Cambiamento di mansioni in altro reparto/ufficio ecc.

- Nessun cambiamento".
} 
Più diffuso è invece l'effetto della conoscenza nelle 'tecnologie abilitanti': la probabilità che si verifichino cambiamenti è infatti positivamente associata al livello medio di conoscenza dichiarato dai partecipanti in tutte le tre circoscrizioni territoriali (la probabilità che si percepiscano cambiamenti all'aumentare di un'unità nel punteggio medio, è maggiore del $10 \%$ al Nord, del $12.8 \%$ al Centro e dell' $11.2 \%$ al Sud). La 'conoscenza tecnologica' sembra quindi influire ovunque sui cambiamenti nell'esercizio del lavoro, diversamente dalla formazione sulle 'innovazioni tecnologiche', che si associa a percezioni soggettive di cambiamenti tra i lavoratori del Nord e del Centro ma non tra quelli delle imprese del Sud.

Tabella 5. Probabilità che si registri almeno un cambiamento nelle mansioni svolte in relazione al livello medio di conoscenza sulle 'tecnologie abilitanti', alle caratteristiche dei lavoratori formati, delle imprese di appartenenza e della formazione fruita

\begin{tabular}{|c|c|c|c|}
\hline Variabili esplicative & Nord & Centro & Sud \\
\hline Formati sulle 'innovazioni tecnologiche' & $\begin{array}{l}0.1065^{* *} \\
{[0.0472]}\end{array}$ & $\begin{array}{c}0.1318 * * * \\
{[0.0484]}\end{array}$ & $\begin{array}{c}0.025 \\
{[0.0484]}\end{array}$ \\
\hline Livello medio di conoscenza sulle 'tecnologie abilitanti' & $\begin{array}{c}0.1047 * * * \\
{[0.0367]}\end{array}$ & $\begin{array}{c}0.1822 * * * \\
{[0.0438]}\end{array}$ & $\begin{array}{l}0.1122 * * \\
{[0.0491]}\end{array}$ \\
\hline \multicolumn{4}{|c|}{ Classi d'età } \\
\hline Da 30 a 49 anni nel confronto con la classe 'fino a 29 anni' & $\begin{array}{c}-0.1346 * * * \\
{[0.0355]}\end{array}$ & $\begin{array}{c}0.0062 \\
{[0.0601]}\end{array}$ & $\begin{array}{c}0.0132 \\
{[0.0443]}\end{array}$ \\
\hline Oltre 50 anni nel confronto con la classe 'fino a 29 anni' & $\begin{array}{c}-0.1978 * * * \\
{[0.0397]}\end{array}$ & $\begin{array}{c}-0.0421 \\
{[0.0657]}\end{array}$ & $\begin{array}{c}0.0621 \\
{[0.0515]}\end{array}$ \\
\hline \multicolumn{4}{|c|}{ Titolo di studio } \\
\hline Diploma nel confronto con la Licenza Media & $\begin{array}{l}-0.0564 \\
{[0.0379]}\end{array}$ & $\begin{array}{l}-0.0361 \\
{[0.0493]}\end{array}$ & $\begin{array}{c}0.0283 \\
{[0.0439]}\end{array}$ \\
\hline Laurea e post-laurea nel confronto con la Licenza Media & $\begin{array}{l}-0.0095 \\
{[0.0492]}\end{array}$ & $\begin{array}{c}0.0256 \\
{[0.0621]}\end{array}$ & $\begin{array}{c}-0.0524 \\
{[0.0697]}\end{array}$ \\
\hline Lavoratrici nel confronto con i lavoratori & $\begin{array}{c}0.0505 \\
{[0.0309]}\end{array}$ & $\begin{array}{l}0.0650 * \\
{[0.0354]}\end{array}$ & $\begin{array}{c}-0.0263 \\
{[0.0405]}\end{array}$ \\
\hline \multicolumn{4}{|c|}{ Inquadramento } \\
\hline Impiegati nel confronto con gli operai & $\begin{array}{c}0.0578 \\
{[0.0458]}\end{array}$ & $\begin{array}{c}-0.1159 * * \\
{[0.0538]}\end{array}$ & $\begin{array}{c}-0.0005 \\
{[0.0512]}\end{array}$ \\
\hline Quadri nel confronto con gli operai & $\begin{array}{c}-0.0166 \\
{[0.0529]}\end{array}$ & $\begin{array}{c}-0.1663^{* *} \\
{[0.0725]}\end{array}$ & $\begin{array}{c}0.1547 \\
{[0.1159]}\end{array}$ \\
\hline \multicolumn{4}{|c|}{ Tipologia contrattuale } \\
\hline Tempo determinato nel confronto con il Tempo Indeterminato & $\begin{array}{l}-0.0304 \\
{[0.0604]}\end{array}$ & $\begin{array}{c}-0.001 \\
{[0.0637]}\end{array}$ & $\begin{array}{c}0.0988 * \\
{[0.0578]}\end{array}$ \\
\hline Part time nel confronto con il Tempo Indeterminato & $\begin{array}{c}0.0933 \\
{[0.1089]}\end{array}$ & $\begin{array}{c}0.0428 \\
{[0.1347]}\end{array}$ & $\begin{array}{c}0.017 \\
{[0.0773]}\end{array}$ \\
\hline Altro contratto nel confronto con il Tempo Indeterminato & $\begin{array}{c}-0.2223^{* *} \\
{[0.0991]}\end{array}$ & $\begin{array}{c}0.141 \\
{[0.2859]}\end{array}$ & $\begin{array}{c}0.4364 * * \\
{[0.2073]}\end{array}$ \\
\hline \multicolumn{4}{|c|}{ Area di attività } \\
\hline Commerciale/marketing & $\begin{array}{l}-0.0428 \\
{[0.0539]}\end{array}$ & $\begin{array}{c}0.0869 \\
{[0.0597]}\end{array}$ & $\begin{array}{c}0.0825 \\
{[0.0908]}\end{array}$ \\
\hline Logistica/magazzino & $\begin{array}{c}0.068 \\
{[0.0574]}\end{array}$ & $\begin{array}{c}0.0757 \\
{[0.0884]}\end{array}$ & $\begin{array}{c}0.0061 \\
{[0.0956]}\end{array}$ \\
\hline Produzione & $\begin{array}{c}0.0396 \\
{[0.0454]}\end{array}$ & $\begin{array}{c}0.0435 \\
{[0.0548]}\end{array}$ & $\begin{array}{c}-0.0117 \\
{[0.0616]}\end{array}$ \\
\hline Ricerca e sviluppo & $\begin{array}{c}0.0364 \\
{[0.0536]}\end{array}$ & $\begin{array}{c}0.0346 \\
{[0.0725]}\end{array}$ & $\begin{array}{c}-0.025 \\
{[0.1126]}\end{array}$ \\
\hline Supporto tecnico/manutenzione & $\begin{array}{c}0.0113 \\
{[0.0539]}\end{array}$ & $\begin{array}{c}0.0524 \\
{[0.0625]}\end{array}$ & $\begin{array}{c}0.0019 \\
{[0.0898]}\end{array}$ \\
\hline Vendita & $\begin{array}{c}0.0706 \\
{[0.0696]}\end{array}$ & $\begin{array}{c}0.0592 \\
{[0.0855]}\end{array}$ & $\begin{array}{c}0.1003 \\
{[0.0975]}\end{array}$ \\
\hline
\end{tabular}




\begin{tabular}{|c|c|c|c|}
\hline Variabili esplicative & Nord & Centro & Sud \\
\hline \multicolumn{4}{|c|}{ Mansioni svolte (nel confronto con la mansione: "Pianificazione e coordinamento delle strategie aziendali") } \\
\hline Analisi di situazioni complesse, sviluppo di nuove conoscenze, diagnosi e ricerca & $\begin{array}{c}-0.1117^{* *} \\
{[0.0505]}\end{array}$ & $\begin{array}{l}-0.0828 \\
{[0.0613]}\end{array}$ & $\begin{array}{c}0.0274 \\
{[0.0909]}\end{array}$ \\
\hline Controllo dei processi di produzione e applicazioni di protocolli operativi & $\begin{array}{c}-0.1011^{* *} \\
{[0.0481]}\end{array}$ & $\begin{array}{c}-0.0691 \\
{[0.0601]}\end{array}$ & $\begin{array}{c}-0.0445 \\
{[0.0796]}\end{array}$ \\
\hline Acquisizione, trattamento, archiviazione e trasmissione delle informazioni & $\begin{array}{l}-0.0755 \\
{[0.0518]}\end{array}$ & $\begin{array}{l}-0.0174 \\
{[0.0598]}\end{array}$ & $\begin{array}{c}-0.1626^{* *} \\
{[0.0808]}\end{array}$ \\
\hline Assistenza ai clienti, ai consumatori, ai cittadini & $\begin{array}{l}-0.1878^{* * *} \\
{[0.0503]}\end{array}$ & $\begin{array}{l}-0.1234^{*} \\
{[0.0638]}\end{array}$ & $\begin{array}{l}-0.1442^{*} \\
{[0.0783]}\end{array}$ \\
\hline Costruzione, riparazione o manutenzione di manufatti, oggetti e macchine & $\begin{array}{l}-0.1255^{* *} \\
{[0.0625]}\end{array}$ & $\begin{array}{l}-0.1104 \\
{[0.0748]}\end{array}$ & $\begin{array}{l}-0.0106 \\
{[0.0921]}\end{array}$ \\
\hline $\begin{array}{l}\text { Conduzione e controllo di macchine industriali e di impianti automatizzati o } \\
\text { robotizzati }\end{array}$ & $\begin{array}{l}-0.2035^{* * *} \\
{[0.0682]}\end{array}$ & $\begin{array}{l}-0.1446^{*} \\
{[0.0829]}\end{array}$ & $\begin{array}{c}-0.2207^{* * *} \\
{[0.0839]}\end{array}$ \\
\hline Compiti di manovalanza in agricoltura edilizia e industria & $\begin{array}{c}-0.2882 * * * \\
{[0.0944]}\end{array}$ & $\begin{array}{c}-0.2023^{* *} \\
{[0.0839]} \\
\end{array}$ & $\begin{array}{c}-0.1867^{* *} \\
{[0.0850]} \\
\end{array}$ \\
\hline \multicolumn{4}{|c|}{ Settore di attività dell'impresa (nel confronto con il settore "Servizi alle imprese") } \\
\hline Industria metalmeccanica & $\begin{array}{c}0.0079 \\
{[0.0375]}\end{array}$ & $\begin{array}{c}-0.0269 \\
{[0.0456]}\end{array}$ & $\begin{array}{c}-0.1179 * * \\
{[0.0499]}\end{array}$ \\
\hline Altra manifattura & $\begin{array}{c}-0.0402 \\
{[0.0387]}\end{array}$ & $\begin{array}{l}-0.0829 * \\
{[0.0429]}\end{array}$ & $\begin{array}{c}-0.0705 \\
{[0.0481]}\end{array}$ \\
\hline Costruzioni e altre industrie non manifatturiera & $\begin{array}{c}-0.1101 \\
{[0.0669]}\end{array}$ & $\begin{array}{c}-0.0098 \\
{[0.0792]}\end{array}$ & $\begin{array}{c}-0.0973 \\
{[0.0660]}\end{array}$ \\
\hline Commercio, alberghi e ristoranti & $\begin{array}{c}0.019 \\
{[0.0500]}\end{array}$ & $\begin{array}{c}-0.0165 \\
{[0.0621]}\end{array}$ & $\begin{array}{c}-0.0115 \\
{[0.0653]}\end{array}$ \\
\hline Sanità, assistenza e servizi alle persone & $\begin{array}{c}-0.03 \\
{[0.0569]}\end{array}$ & $\begin{array}{c}0.1363^{* *} \\
{[0.0666]} \\
\end{array}$ & $\begin{array}{c}0.0535 \\
{[0.0622]} \\
\end{array}$ \\
\hline \multicolumn{4}{|l|}{ Classi dimensionali dell'impresa } \\
\hline Da 50 a 249 dipendenti nel confronto con le imprese con meno di 50 & $\begin{array}{c}0.1068^{* * *} \\
{[0.0294]}\end{array}$ & $\begin{array}{c}0.037 \\
{[0.0336]}\end{array}$ & $\begin{array}{c}-0.0509 \\
{[0.0378]}\end{array}$ \\
\hline Oltre 250 dipendenti nel confronto con le imprese con meno di 50 & $\begin{array}{c}0.1498 * * * \\
{[0.0330]}\end{array}$ & $\begin{array}{c}0.1150 * * * \\
{[0.0426]}\end{array}$ & $\begin{array}{c}0.0002 \\
{[0.0582]}\end{array}$ \\
\hline Durata della formazione & $\begin{array}{c}0.0021 * * * \\
{[0.0007]}\end{array}$ & $\begin{array}{c}0.0015^{* *} \\
{[0.0006]}\end{array}$ & $\begin{array}{c}0.0002 \\
{[0.0007]}\end{array}$ \\
\hline \multicolumn{4}{|c|}{ Condivisione dell'esperienza formativa con il responsabile (nel confronto con la completa assenza di condivisione) } \\
\hline Prima della formazione & $\begin{array}{l}0.0824 * \\
{[0.0443]}\end{array}$ & $\begin{array}{c}0.0812 \\
{[0.0596]}\end{array}$ & $\begin{array}{c}0.0079 \\
{[0.0724]}\end{array}$ \\
\hline Dopo la formazione & $\begin{array}{c}0.1157^{* * *} \\
{[0.0407]}\end{array}$ & $\begin{array}{c}0.0609 \\
{[0.0502]}\end{array}$ & $\begin{array}{c}0.0361 \\
{[0.0725]}\end{array}$ \\
\hline Sia prima che dopo & $\begin{array}{c}0.0701^{* *} \\
{[0.0348]}\end{array}$ & $\begin{array}{c}0.0654 \\
{[0.0445]}\end{array}$ & $\begin{array}{c}0.0361 \\
{[0.0655]}\end{array}$ \\
\hline $\begin{array}{l}\text { Finanziamento dell'iniziativa con il "Conto Formazione" nel confronto con il } \\
\text { "Conto di Sistema" }\end{array}$ & $\begin{array}{c}0.0447 \\
{[0.0302]}\end{array}$ & $\begin{array}{c}0.014 \\
{[0.0361]}\end{array}$ & $\begin{array}{l}-0.0107 \\
{[0.0455]}\end{array}$ \\
\hline $\begin{array}{l}\text { Iniziative formative maggiormente coerenti alle mansioni svolte in impresa } \\
\text { (nella percezione del lavoratore) }\end{array}$ & $\begin{array}{l}0.1160^{*} \\
{[0.0663]}\end{array}$ & $\begin{array}{l}-0.0752 \\
{[0.0707]}\end{array}$ & $\begin{array}{c}0.0728 \\
{[0.0741]}\end{array}$ \\
\hline Formazione realizzata anche con metodologie diverse dall'aula & $\begin{array}{l}0.0764^{*} \\
{[0.0450]}\end{array}$ & $\begin{array}{c}-0.005 \\
{[0.0518]}\end{array}$ & $\begin{array}{l}-0.0338 \\
{[0.0676]}\end{array}$ \\
\hline Totale osservazioni $(N)$ & 2452 & 1436 & 1447 \\
\hline
\end{tabular}

Nota: i valori assumono valore positivo o negativo a seconda del segno della relazione con la variabile esplicativa (diretta o inversa). Gli asterischi indicano tre diversi livelli di significatività: ${ }^{* *} p<0.01, * * p<0.05, * p<0.1$.

Fonte: nostre elaborazioni su dati Indagine ROLA 2019, Fondimpresa

L'assenza di effetti sulla percezione dei cambiamenti tra i lavoratori del Sud riguarda, del resto, quasi tutte le variabili esplicative utilizzate: le sole che sembrano raggiungere adeguati livelli di significatività sono quelle relative alla tipologia contrattuale e al grado di complessità delle mansioni svolte in 
azienda. Si tratta comunque di aspetti marginali (oltretutto non sufficientemente chiari) nel primo caso e di relazioni piuttosto ovvie nel secondo.

Per quanto riguarda in particolare la tipologia contrattuale, si osserva una maggiore probabilità che si registrino cambiamenti nelle mansioni tra i lavoratori inquadrati con contratti di lavoro diversi dal Tempo Indeterminato e, in misura ancora maggiore, tra quelli con contratti diversi anche dal Tempo Determinato, definiti come 'altri contratti'. In attesa di ulteriori approfondimenti, che saranno realizzati nelle prossime edizioni dell'indagine ROLA, un impatto così forte nella percezione dei formati (43 p.p. in più rispetto ai lavoratori con contratto a TI) sembrerebbe caratteristico degli effetti del cambiamento di status dei disoccupati dopo una prima formazione propedeutica all'ingresso in un nuovo lavoro, oppure della formazione degli apprendisti o dei tirocinanti.

Per quanto concerne invece la relazione tra la percezione del cambiamento e il grado di complessità dei compiti di lavoro, ovunque, al decrescere di questa, si registra una diminuzione delle probabilità di registrare cambiamenti delle mansioni a seguito della formazione. Si tratta di una relazione frequentemente osservata nella maggioranza dei riscontri empirici e trova conferma anche in questo caso: più i compiti sono elementari, meno sono suscettibili di sviluppo e riqualificazione attraverso ordinari interventi formativi, a meno che non vengano trasformati (in genere radicalmente) dall'introduzione di nuove tecnologie.

Sensibilmente più articolato è il quadro del problema al Centro e al Nord: oltre al grado di complessità dei compiti, svolgono un ruolo significativo nella percezione del cambiamento le caratteristiche demografiche dei formati (maggiori cambiamenti tra i più giovani), la dimensione dell'impresa di appartenenza (cresce la percezione di cambiamenti al crescere di questa) ma, soprattutto, le caratteristiche dell'iniziativa formativa tra le quali emerge, come spesso avviene e come già sottolineato, la condivisione dell'esperienza con il proprio responsabile aziendale, ma anche il grado di coerenza tra il lavoro e i contenuti della formazione e la diversificazione nelle metodologie utilizzate.

Questo legame, che si osserva in particolare al Nord, tra i caratteri e le peculiarità della formazione e le ricadute di questa in termini di mutamenti nell'esercizio del lavoro, rappresenta la connessione più logica e diretta la cui presenza e intensità è indispensabile per misurare il grado di efficacia dell'intero processo. In questo senso, la mancanza di connessione, osservata tra i lavoratori formati del Sud, tra formazione sulle 'innovazioni tecnologiche' e cambiamenti nelle mansioni può essere meglio compresa: essa sussiste e agisce quanto più l'intervento formativo è progettato e realizzato in stretta relazione con le reali esigenze e allo stesso tempo, quanto più l'impresa è nelle condizioni di tradurre efficacemente gli esiti in comportamenti e azioni. A loro volta, come ovvio, tali elementi dipendono dall'operare di numerosi e complessi meccanismi, il governo e il controllo dei quali richiede condizioni di contesto adatte. $\mathrm{Ci}$ si riferisce in particolare alla possibilità di condurre efficaci analisi dei fabbisogni di competenze (e quindi dell'esistenza di professionalità adeguate o di servizi di assistenza forniti dal sistema pubblico o dalla bilateralità, in particolare per le piccole imprese), all'expertise delle strutture dell'offerta formativa, al posizionamento competitivo delle imprese coinvolte. Si tratta, in sostanza, di tutto quel complesso di caratteristiche ed elementi che connotano e definiscono il livello di evoluzione e di sviluppo socioeconomico di un contesto territoriale, di cui il sistema formativo non è che un coerente sottoinsieme.

Allo scopo di accertare la robustezza di quanto evidenziato nell'analisi, ed in particolare la mancata percezione di cambiamenti nel lavoro tra i formati del Mezzogiorno sui temi dell'innovazione tecnologica', si è proceduto, applicando la medesima metodologia, ad una verifica degli effetti tra i 
lavoratori coinvolti in iniziative formative centrate su tematiche diverse e più generali riguardanti in particolare lo "sviluppo delle capacità personali e la conoscenza del contesto lavorativo". In quest'ambito tematico vengono solitamente raggruppati tutti gli argomenti affrontati nella formazione generale in ingresso e nella "prima formazione al ruolo".

Il test così condotto ha quindi lo scopo:

- di rilevare l'esistenza di diversi effetti sulla percezione dei cambiamenti al variare delle tematiche formative;

- di escludere che, tra i lavoratori del Mezzogiorno, vi sia una generalizzata indifferenza nella percezione del cambiamento a prescindere dai temi trattati nella formazione.

Come illustrato nella seguente tabella 6 , i lavoratori formati sullo "sviluppo delle abilità personali e la conoscenza del contesto lavorativo" mostrano una probabilità maggiore di percepire cambiamenti, rispetto ai formati su altri temi, pari a 10.3 punti percentuali al Centro, 11.6 al Nord e 13.4 al Sud. Ciò consente di escludere categoricamente l'esistenza di una resistenza generalizzata alla percezione del cambiamento tra i lavoratori del Mezzogiorno: al contrario, tra i formati su tematiche più generali, gli effetti al Sud sono superiori di quelli riscontrati sia al Nord, sia al Centro.

Tabella 6. Probabilità che si registri almeno un cambiamento nelle mansioni svolte tra chi ha frequentato un corso in materia di 'Sviluppo delle capacità personali' e in relazione alla tipologia contrattuale, alla durata della formazione e alle mansioni svolte ordinariamente in azienda. Modello probit, effetti medi marginali

\begin{tabular}{|c|c|c|c|}
\hline \multirow[t]{2}{*}{ Variabili esplicative } & \multicolumn{3}{|c|}{$\begin{array}{l}\text { Probabilità che si percepiscano } \\
\text { cambiamenti delle mansioni svolte }\end{array}$} \\
\hline & Nord & Centro & Sud \\
\hline Formati sullo Sviluppo delle abilità personali e conoscenza del contesto lavorativo & $\begin{array}{c}0.1164 * * * \\
{[0.0274]}\end{array}$ & $\begin{array}{c}0.1026^{* * *} \\
{[0.0394]}\end{array}$ & $\begin{array}{c}0.1346^{* * *} \\
{[0.0428]}\end{array}$ \\
\hline \multicolumn{4}{|l|}{ Tipologia contrattuale } \\
\hline Tempo determinato nel confronto con il Tempo Indeterminato & $\begin{array}{l}-0.0253 \\
{[0.0601]}\end{array}$ & $\begin{array}{c}0.0092 \\
{[0.0667]}\end{array}$ & $\begin{array}{c}0.0929 \\
{[0.0575]}\end{array}$ \\
\hline Altro contratto nel confronto con il Tempo Indeterminato & $\begin{array}{c}-0.2186 * * \\
{[0.1043]}\end{array}$ & $\begin{array}{c}0.1471 \\
{[0.2980]}\end{array}$ & $\begin{array}{c}0.4397^{* *} \\
{[0.2027]}\end{array}$ \\
\hline Durata della formazione & $\begin{array}{c}0.0023^{* * *} \\
{[0.0007]}\end{array}$ & $\begin{array}{c}0.0019 * * * \\
{[0.0007]}\end{array}$ & $\begin{array}{c}0.0005 \\
{[0.0007]}\end{array}$ \\
\hline \multicolumn{4}{|l|}{ Mansioni svolte } \\
\hline Analisi di situazioni complesse, sviluppo di nuove conoscenze, diagnosi e ricerca & $\begin{array}{l}-0.1006^{*} \\
{[0.0513]}\end{array}$ & $\begin{array}{l}-0.0533 \\
{[0.0645]}\end{array}$ & $\begin{array}{c}0.0028 \\
{[0.0890]}\end{array}$ \\
\hline Controllo dei processi di produzione e applicazioni di protocolli operativi & $\begin{array}{l}-0.0943 * \\
{[0.0484]}\end{array}$ & $\begin{array}{l}-0.0533 \\
{[0.0616]}\end{array}$ & $\begin{array}{l}-0.0775 \\
{[0.0794]}\end{array}$ \\
\hline Acquisizione, trattamento, archiviazione e trasmissione delle informazioni & $\begin{array}{l}-0.0787 \\
{[0.0524]}\end{array}$ & $\begin{array}{c}-0.007 \\
{[0.0619]}\end{array}$ & $\begin{array}{c}-0.1777^{* *} \\
{[0.0783]}\end{array}$ \\
\hline Assistenza ai clienti, ai consumatori, ai cittadini & $\begin{array}{c}-0.1883 * * * \\
{[0.0501]}\end{array}$ & $\begin{array}{l}-0.1021 \\
{[0.0672]}\end{array}$ & $\begin{array}{c}-0.1676^{* *} \\
{[0.0784]}\end{array}$ \\
\hline Costruzione, riparazione o manutenzione di manufatti, oggetti e macchine & $\begin{array}{l}-0.1185^{*} \\
{[0.0625]}\end{array}$ & $\begin{array}{c}-0.105 \\
{[0.0790]}\end{array}$ & $\begin{array}{l}-0.0479 \\
{[0.0890]}\end{array}$ \\
\hline $\begin{array}{l}\text { Conduzione e controllo di macchine industriali e di impianti automatizzati o } \\
\text { robotizzati }\end{array}$ & $\begin{array}{l}-0.1768 * * * \\
{[0.0685]}\end{array}$ & $\begin{array}{c}-0.108 \\
{[0.0886]}\end{array}$ & $\begin{array}{l}-0.2150 * * * \\
{[0.0820]}\end{array}$ \\
\hline Compiti di manovalanza in agricoltura edilizia e industria & $\begin{array}{c}-0.2878 * * * \\
{[0.0946]}\end{array}$ & $\begin{array}{l}-0.1860 * * \\
{[0.0865]}\end{array}$ & $\begin{array}{c}-0.2152^{* *} \\
{[0.0836]}\end{array}$ \\
\hline
\end{tabular}

Nota: i valori assumono valore positivo o negativo a seconda del segno della relazione con la variabile esplicativa (diretta o inversa). Gli asterischi indicano tre diversi livelli di significatività: ${ }^{* * *} p<0.01,{ }^{* *} p<0.05,{ }^{*} p<0.1$.

Fonte: nostre elaborazioni su dati Indagine ROLA 2019, Fondimpresa 
L'assenza di cambiamenti percepiti riguarda quindi, effettivamente, i lavoratori del Sud formati sui temi dell'innovazione tecnologica' e su tale particolare relazione dovrà essere concentrato lo sforzo interpretativo.

L'attendibilità del test trova peraltro conferma anche nell'emergere delle medesime relazioni evidenziate nell'analisi principale: ci si riferisce alla relazione inversa già osservata tra gli effetti sui cambiamenti e la minore complessità delle mansioni svolte e di quella, del tutto peculiare, relativa alle tipologie contrattuali atipiche (da approfondire, come già accennato, nelle prossime edizioni dell'indagine).

È molto utile verificare, infine, quanto i livelli di conoscenza dichiarati su ognuna delle sei 'tecnologie abilitanti' possano condizionare le probabilità che i lavoratori formati riscontrino cambiamenti nelle mansioni esercitate in azienda. A tal fine, utilizzando medesima metodologia delle precedenti elaborazioni, è stato attribuito un valore pari a 0 a chi non ha fatto formazione sulle 'innovazioni tecnologiche' e la differenza di punteggio dei livelli di conoscenza sulle 'tecnologie abilitanti', registrati prima e dopo il corso, a coloro che hanno invece fruito della formazione sulle 'innovazioni'. Verifichiamo, quindi, se in ciascuna delle tre macroaree territoriali l'ampiezza della differenza tra i livelli di conoscenza registrati prima e dopo la formazione, per ciascuna tecnologia abilitante, favorisca o meno la probabilità che si percepiscano cambiamenti.

Come prevedibile, ciò si riscontra quasi sempre, seppure con gradi di intensità diversi (tabella 7) e questo rappresenta, ai nostri fini, il punto essenziale. Oltre alla conferma di una relazione generale, emergono effetti fortemente differenziati per alcuni cluster tecnologici che, a loro volta, si declinano con particolare evidenza sul piano territoriale con modalità più articolate di quanto ci si potrebbe attendere.

Tabella 7. Probabilità che si registri almeno un cambiamento nelle mansioni svolte in relazione all'ampiezza della differenza dei livelli di conoscenza percepiti dai lavoratori su ciascuna 'tecnologia abilitante' prima e dopo la formazione sulle 'innovazioni tecnologiche'. Modello probit, effetti medi marginali

\begin{tabular}{lccc}
\hline \multirow{2}{*}{ Tecnologie abilitanti } & Probabilità che si percepiscano cambiamenti delle mansioni svolte \\
\cline { 2 - 4 } & Nord & Centro & Sud \\
\hline Industrial Internet of Things & $-0.1273^{*}$ & 0.0517 & -0.101 \\
& {$[0.0769]$} & {$[0.0926]$} & {$[0.0690]$} \\
Industrial Analytics (Big Data) & 0.054 & $0.1770^{* *}$ & 0.0397 \\
& {$[0.0615]$} & {$[0.0759]$} & {$[0.0874]$} \\
Cloud Manufacturing & $0.1947^{* * *}$ & 0.1048 & 0.0757 \\
& {$[0.0638]$} & {$[0.0773]$} & {$[0.0878]$} \\
Advanced Automation (Robotica collaborativa) & -0.0883 & -0.0832 & $0.2777^{* * *}$ \\
Advanced Human Machine Interface (HMI) & {$[0.0844]$} & {$[0.0796]$} & {$[0.0952]$} \\
Additive Manufacturing & 0.0072 & 0.1107 & -0.0771 \\
& {$[0.0859]$} & {$[0.0896]$} & {$[0.0887]$} \\
\hline
\end{tabular}

Nota: i valori sono compresi tra 1 e 0 . Assumono valore positivo o negativo a seconda del segno della relazione con la variabile esplicativa (diretta o inversa). Gli asterischi indicano tre diversi livelli di significatività: *** $p<0.01, * * p<0.05,{ }^{*} p<0.1$.

Fonte: nostre elaborazioni su dati Indagine ROLA 2019, Fondimpresa 
Si tratta di indicazioni importanti su quello che potrebbe essere stato il livello di inerenza e di efficacia della formazione sui temi dell'innovazione tecnologica' nei diversi ambiti territoriali. In altri termini, ad esempio, parte della formazione sull'innovazione realizzata al Sud ha contribuito all'accrescimento dei livelli di conoscenza sul cluster della Robotica collaborativa ed ha prodotto percepibili cambiamenti nelle mansioni svolte. Stesse considerazioni per quanto riguarda il Cloud Manufacturing al Nord, I'Industrial Analytics e l'HMI al Centro. Come ovvio, maggiore importanza interpretativa va attribuita agli effetti della formazione piuttosto che alla caratterizzazione territoriale in sé considerata, che può dipendere anche dalle specifiche localizzazioni di distretti produttivi tecnologicamente specializzati (tenendo conto peraltro che, per le nuove tecnologie digitali, le specificità territoriali tradizionali hanno un'importanza sensibilmente minore rispetto al passato).

Diversamente, l'accrescimento della conoscenza connesso alla formazione 'sulle innovazioni' per quanto riguarda in particolare l'Additive Manufacturing al Sud e l'Internet of Things al Nord e al Sud, sembra invece aver avuto una relazione inversa con la percezione del cambiamento di mansioni. La probabile presenza di interventi formativi che hanno dispiegato i loro effetti sull'accrescimento delle conoscenze ma che ritardano a manifestare percepibili ricadute sull'esercizio del lavoro, non è quindi caratterista esclusiva del Sud ma, in certi ambiti e a certe condizioni, sembra potersi osservare anche in altre aree del Paese.

\section{Conclusioni}

Alla formazione sui temi dell'innovazione tecnologica, corrisponde con sistematicità, tra i lavoratori formati, la percezione di una crescita dei livelli di conoscenza sui cluster tecnologici di maggiore rilevanza strategica (le sei tecnologie abilitanti qui considerate). Ciò si verifica in tutti gli ambiti territoriali, seppure in modo relativamente più contenuto tra i formati del Mezzogiorno.

Diversamente, la percezione di cambiamenti nelle mansioni svolte, connessi con la formazione sui medesimi temi, risulta presente tra i formati del Nord e del Centro ma non tra i lavoratori del Sud.

Ciò non rappresenta una condizione eccezionale: come già osservato, le due dinamiche possono coesistere e procedere in modo disgiunto. All'accrescimento delle conoscenze prodotto dalla formazione non sempre corrisponde una concreta e uniforme ricaduta nell'esercizio del lavoro. Non sussiste una relazione necessaria: tra $\mathrm{i}$ due aspetti possono frapporsi numerose e articolate circostanze e condizioni di contesto.

La connessione tra la percezione dei cambiamenti nel lavoro e la formazione fruita, anche nei casi in cui essa abbia prodotto positivi ed evidenti effetti sui livelli di conoscenza, dipende da un numero molto elevato di variabili, alcune controllabili con buoni margini di manovra, altre, di natura strutturale, le cui dinamiche di mutamento possono essere lunghe e complesse e sostanzialmente fuori dalla portata delle organizzazioni coinvolte nei processi di promozione, finanziamento e realizzazione della formazione

Tra le variabili maggiormente suscettibili di intervento vanno considerate gli elementi propri delle iniziative formative ed in particolare: I'aderenza degli interventi con i compiti effettivamente svolti dai lavoratori; le caratteristiche dei partecipanti (e quindi i criteri di scelta e selezione di questi); le modalità di realizzazione della formazione e le metodologie utilizzate. Proprio su questi punti l'analisi ha consentito di evidenziare con chiarezza come una delle maggiori difformità tra i territori (tra il 
Centro-Nord e il Sud) riguardasse proprio alcuni elementi fondamentali connessi con la realizzazione concreta delle iniziative, tra i quali è emersa la condivisione dell'esperienza con il proprio responsabile aziendale, ma anche la diversificazione nelle metodologie utilizzate (migliori risultati sono connessi all'uso di metodologie esperienziali) e il grado di coerenza tra il lavoro concretamente svolto e i contenuti della formazione.

Abbiamo osservato, quindi, come il legame tra i caratteri della formazione e le ricadute di questa in termini di mutamenti nell'esercizio del lavoro (che si osserva al Nord e al Centro), potrebbe rappresentare una delle possibili chiavi di lettura e come però emerga, parallelamente, l'importanza di una concreta e operante connessione tra le peculiarità della domanda e dell'offerta di formazione a valle del processo. Ci si riferisce in particolare al grado di utilizzo delle nuove competenze dei formati che l'impresa può effettivamente conseguire (e in che tempi ciò possa avvenire, rispetto ai tempi della formazione e alle aspettative dei partecipanti) e ancora, più in generale, alla capacità effettiva di impiego e di mobilitazione di queste nella realtà del processo produttivo. Ciò è strettamente legato alla possibilità di condurre efficaci analisi dei fabbisogni di competenze (e quindi dell'esistenza di professionalità adeguate o di servizi di assistenza forniti dal sistema pubblico o dalla bilateralità, in particolare per le piccole imprese), all'expertise delle strutture dell'offerta formativa, al posizionamento competitivo delle imprese coinvolte. Si tratta precisamente di quelle condizioni di contesto sulle quali i margini di intervento sono molto minori.

Un sistema formativo non è che un sottoinsieme coerente di un determinato e più ampio contesto socioeconomico con il quale condivide il livello di evoluzione e di sviluppo.

L'organizzazione di Fondimpresa e l'insieme delle pratiche formative promosse negli anni tra le sue aziende aderenti, rappresenta probabilmente una delle parti del sistema nazionale di formazione continua più avanzata ed efficiente. Ciò non è tuttavia sufficiente a superare d'un tratto le difficoltà che, nel Mezzogiorno, la formazione sui temi dell'innovazione incontra nel produrre effetti paragonabili a quelli invece facilmente conseguibili al Nord e al Centro.

II minore sviluppo tecnologico delle imprese meridionali, induce spesso le strutture locali dell'offerta formativa a seguire una linea basata maggiormente su interventi di primo approccio alle tecnologie che, pur sviluppando tra i formati la percezione di un aumento anche significativo delle conoscenze (come evidenziato dai dati qui illustrati), solo sporadicamente viene poi tradotto in operatività e concretamente agito. Nella maggioranza dei casi, potrebbe infatti non esservi stato un effettivo training sull'utilizzo diretto (che a sua volta presupporrebbe strutture di formazione parimenti tecnologicamente dotate) oppure l'utilizzo della particolare tecnologia oggetto della formazione, potrebbe non essere adeguatamente diffuso e, soprattutto, realmente assimilato nei processi produttivi delle imprese partecipanti.

D'altra parte, è anche necessario tener presente che le iniziative dirette al primo approccio e maggiormente indirizzate alla diffusione della conoscenza, piuttosto che all'addestramento concreto al suo utilizzo, oltre che rappresentare la strada di minor resistenza per l'operatività di un sistema formativo fortemente legato ai settori tradizionali, potrebbero essere in qualche caso anche le più adatte ad una forza lavoro ancora parzialmente coinvolta sui segmenti più avanzati dell'innovazione e nei casi in cui l'obiettivo fosse proprio la sensibilizzazione e l'introduzione ai temi. Un esempio particolarmente calzante in questo senso è fornito dalle tematiche ambientali e dagli Avvisi Ambiente e Territorio promossi da Fondimpresa con un accentuato impegno proprio nel Mezzogiorno. In questo caso, il diverso posizionamento delle imprese del Centro e del Nord rispetto a quelle del Sud sui temi 
ambientali, giustifica largamente un approccio formativo più generalista, sia in fase progettuale, sia in fase realizzativa, perché più adeguato al target. Le tecnologie verdi sono del resto il tipico ambito in cui, coerentemente con quanto osservato, le attività di sensibilizzazione dirette alla maturazione della consapevolezza e all'orientamento dei comportamenti, possono ben avere maggiore impatto sulla percezione soggettiva dell'accrescimento di conoscenza, mentre molto minore può essere l'effetto sui reali cambiamenti nelle mansioni.

Per quanto le dinamiche dei Fondi Interprofessionali tendano alla valorizzazione delle scelte delle singole imprese, che sono quindi poste in grado di selezionare l'offerta di formazione che preferiscono (e per quanto ciò stesso possa favorire un meccanismo virtuoso di selezione qualitativa), il ricorso alle Agenzie formative attive sui territori sarà sempre determinante per quella importante quota di attività finanziata con gli Avvisi e diretta prevalentemente alle piccole e piccolissime imprese (Ferri e Tesauro 2018). Solo le strutture dell'offerta formativa, integrate nei diversi contesti (e con le quali le imprese hanno lunga consuetudine) sono in grado di rispondere con la necessaria efficienza e rapidità agli Avvisi diretti al finanziamento dei grandi Piani territoriali, settoriali, di filiera, o dedicati ad altri gruppi/target, aggregando e organizzando la domanda delle aziende e costituendo concretamente i gruppi classe. Peraltro, come evidenziato nell'analisi, sono proprio le iniziative mutualistiche promosse con gli Avvisi quelle che hanno prodotto effetti di assoluto rilievo nella percezione dell'accrescimento dei livelli di 'conoscenza tecnologica' dei partecipanti.

Allo scopo di tradurre ciò in cambiamento effettivo nella quotidianità dell'esercizio delle mansioni di lavoro, possono contribuire almeno in parte le indicazioni operative fornite dalle evidenze qui illustrate, che sono essenziali ma estremamente chiare su almeno tre punti, tra loro connessi:

- la progettazione formativa in tema di innovazione deve svolgersi con il coinvolgimento attivo delle figure di responsabilità e coordinamento che operano concretamente a stretto contatto con i lavoratori da avviare in formazione, assicurando che ciò avvenga in tutte le fasi, dalla definizione e focalizzazione degli obiettivi al follow up;

- l'obiettivo dell'iniziativa deve essere preciso, verificabile e non generico, non può limitarsi alla definizione degli obiettivi di apprendimento ma, con il concorso del responsabile aziendale, deve definire con esaustività le nuove funzioni e mansioni che il lavoratore formato dovrà presidiare;

- la metodologia formativa deve essere definita in coerenza con le reali esigenze, evitando quanto possibile le posizioni preconcette che hanno condizionato il dibattito recente. In questo senso, non sempre il learning by doing è adatto, non sempre le ore d'aula vanno limitate. Uno dei vantaggi dell'introduzione delle nuove tecnologie digitali consiste proprio nella messa in discussione di formule di comodo.

Maggiori elementi per l'interpretazione dei fenomeni trattati in questo studio, saranno assicurati dai risultati della prossima edizione dell'indagine ROLA: grazie ad una relazione molto più stretta tra i contenuti della formazione fruita e le 'tecnologie abilitanti', la rilevazione delle percezioni dei formati, sia in termini di livelli di conoscenza, sia per quanto riguarda i cambiamenti nelle mansioni di lavoro, potrà essere più puntuale. 


\section{Bibliografia}

Abadi F. Perkasa D.H. (2020), Analysis of Job Training, Compensation and Engagement on Job Effectiveness, in $4^{\text {th }}$ International Conference on Management, Economics and Business (ICMEB 2019), Dordrecht-Paris-Zhengzhou, Atlantis Press, pp.191-195

Bachmann R., Cim M., Green C. (2018), Long-run Patterns of Labour Market Polarization. Evidence from German Micro Data, British Journal of Industrial Relations, 57, n.2, pp.350-376

Fondimpresa, Inapp, Bernava M., Ferri V. (a cura di) (2020), L'impatto della formazione continua in azienda. Rapporto ROLA 2019. Rilevazione delle Opinioni dei Lavoratori in Azienda, Roma, Fondimpresa-Inapp <https://bit.ly/3BPir5v>

Brynjolfsson E., Mitchell T., Rock D. (2018), What Can Machines Learn, and What Does It Mean for Occupations and the Economy?, AEA Papers and Proceedings, 108, pp.43-47

Damei M.Y. (2020), The Effect of Job Training on Employee Retention in Somalia, Quest Journals, Journal of Research in Business and Management, 8, n.1, pp.9-17

Ferri V., Tesauro G. (2018), I Fondi Interprofessionali nella strategia d'impresa, Sinappsi, VIII, n.1, pp.49-59

Ferri V., Ricci A. (2020), Investimenti e domanda di profili professionali. Evidenze dalle imprese italiane, Rivista Italiana di Economia Demografia e Statistica, LXXIV, n.3-4, pp.99-110

Ferri V., Guarascio D., Ricci A. (2017), Formazione professionale, innovazione e investimenti in capitale fisico. Evidenze empiriche dei dati RIL, Inapp Paper n.6, Roma, Inapp

Görlitz K., Tamm M. (2016), The returns to voucher-financed training on wages, employment and job tasks, Economics of Education Review, 52, issue C, pp.51-62

Kashif A.R., Shafiq M., Tahir A.H., Wahid S., Ahmed S. (2020), Impact of On Job Training, Training Design and Training Delivery Style on Organizations Performance, FUJBE, 5, n.2, pp.40-51

Lukowski F., Baum M., Mohr S. (2020), Technology, tasks and training - Evidence on the provision of employer-provided training in times of technological change in Germany, Studies in Continuing Education, 43, n.3, pp.1-22

Marwan B.I., Rokaya A. (2020), The Impact of Training on the Relationship between the Person-Job Fit and Employees' Job Engagement, International Journal of Advanced Science and Technology, 29, n.7, pp.13269-13286

Mohr S., Troltsch K., Gerhards C. (2016), Job tasks and the participation of low-skilled employees in employer-provided continuing training in Germany, Journal of Education and Work, 29, n.5, pp.562-583

Tamm M. (2018), Training and changes in job tasks, IZA Discussion Paper n.11787, Bonn, IZA <https://bit.ly/3BQbtZP> 


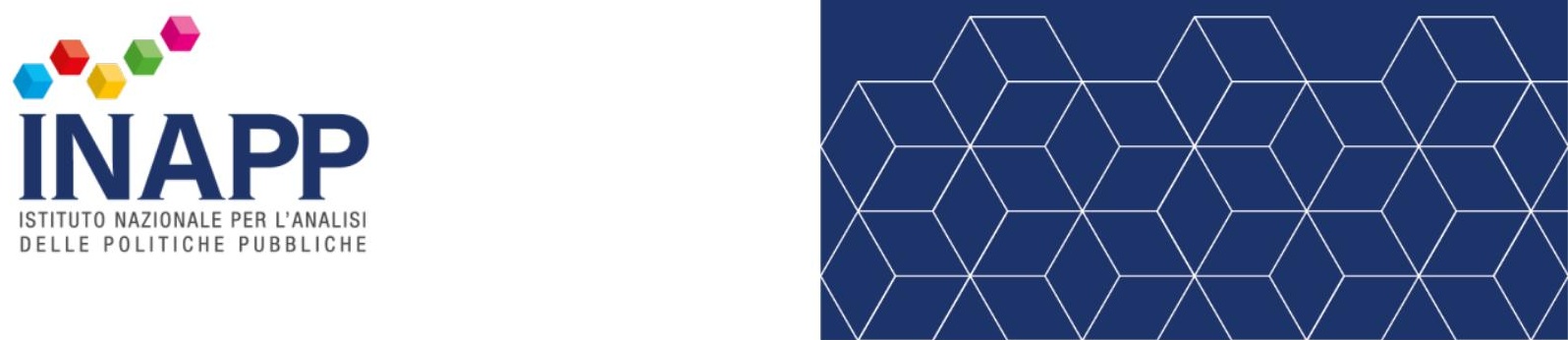